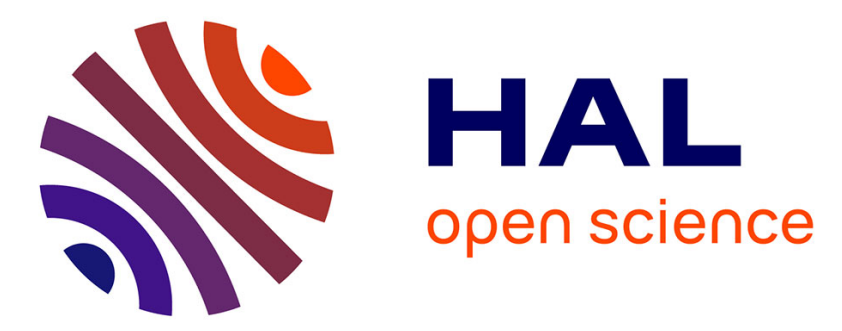

\title{
Collective memory shapes the organization of individual memories in the medial prefrontal cortex
}

Pierre Gagnepain, Thomas Vallée, Serge Heiden, Matthieu Decorde, Jean-Luc

Gauvain, Antoine Laurent, Carine Klein-Peschanski, Fausto Viader, Denis

Peschanski, Francis Eustache

\section{To cite this version:}

Pierre Gagnepain, Thomas Vallée, Serge Heiden, Matthieu Decorde, Jean-Luc Gauvain, et al.. Collective memory shapes the organization of individual memories in the medial prefrontal cortex. Nature Human Behaviour, 2019, 10.1038/s41562-019-0779-z . halshs-02416130

\section{HAL Id: halshs-02416130 \\ https://shs.hal.science/halshs-02416130}

Submitted on 20 Feb 2020

HAL is a multi-disciplinary open access archive for the deposit and dissemination of scientific research documents, whether they are published or not. The documents may come from teaching and research institutions in France or abroad, or from public or private research centers.
L'archive ouverte pluridisciplinaire HAL, est destinée au dépôt et à la diffusion de documents scientifiques de niveau recherche, publiés ou non, émanant des établissements d'enseignement et de recherche français ou étrangers, des laboratoires publics ou privés. 
Gagnepain, P., Vallée, T., Heiden, S. et al. Collective memory shapes the organization of individual memories in the medial prefrontal cortex. Nat Hum Behav 4, 189-200 (2020).

https://doi.org/10.1038/s41562-019-0779-z

https://www.nature.com/articles/s41562-019-0779-z 


\title{
Collective memory shapes the organization of individual memories in the medial prefrontal cortex
}

\author{
Pierre Gagnepain ${ }^{1 *}$, Thomas Vallée ${ }^{1}$, Serge Heiden ${ }^{2}$, Matthieu Decorde ${ }^{2}$, Jean-Luc Gauvain ${ }^{3}$, Antoine \\ Laurent $^{3,4}$, Carine Klein-Peschanski ${ }^{5}$, Fausto Viader ${ }^{1}$, Denis Peschanski ${ }^{5}$ and Francis Eustache ${ }^{1}$
}

It has long been hypothesized that individual recollection fits collective memory. To look for a collective schema, we analysed the content of $\mathbf{3 0}$ years of media coverage of World War II on French national television. We recorded human brain activity using functional magnetic resonance imaging as participants recalled World War II displays at the Caen Memorial Museum following an initial tour. We focused on the medial prefrontal cortex, a key region for social cognition and memory schemas. The organization of individual memories captured using the distribution of the functional magnetic resonance imaging signal in the dorsal part of the medial prefrontal cortex was more accurately predicted by the structure of the collective schema than by various control models of contextual or semantic memory. Collective memory, which exists outside and beyond individuals, can also organize individual memories and constitutes a common mental model that connects people's memories across time and space.

Almost 100 years ago, it was hypothesized that individual memory reconstructs past experiences on the basis of social frameworks bound to collective memory ${ }^{1}$. According to this view, collective memory supplies a long-term organizational model (or schema) of historical and social knowledge, symbols, narratives and images, into which individual memory must fit ${ }^{2}$. Collective representations of the past are selected according to schematic narrative templates, often emphasizing some elements and minimizing others, that define the group's identity ${ }^{3}$. These collective representations are stored both within individuals connected by a common historical past as shared memories, and outside individuals through sociocultural means such as the media ${ }^{4-6}$.

Until now, research in cognitive neuroscience and psychology has adopted a bottom-up approach to highlight the individual cognitive mechanisms that underpin the formation of shared knowledge about collective events ${ }^{7,8}$. The top-down approach (that is, how cultures, mediated by several different resources, shape and influence individuals' cognitive structures, ${ }^{6,9}$ ) has received less attention. The notion of memory schemas, put forward by Bartlett ${ }^{2}$ and later developed by neuroscientists (see Gilboa and Malartte ${ }^{10}$ for a recent review), together with the concept of social knowledge ${ }^{11,12}$, provides a conceptual and neurobiological framework to account for the influence of preexisting collective knowledge on the retrieval of declarative memory. Here, we tested the hypothesis that memory representations in the medial prefrontal cortex (mPFC), whose role in representing memory schemas and social knowledge has been clearly established ${ }^{10-12}$, are also organized by collective schemas acquired in the course of a lifetime.

This hypothesis was based on two distinct lines of research that associate the functioning of the ventral ( $\mathrm{vmPFC}$ ) and dorsal (dmPFC) portions of the mPFC with schema processing and social knowledge, respectively. The first line of research involves brain imaging studies on the vmPFC, which have shown that this structure supports the formation of elaborate neural networks that organize abstract information and concepts into schemas ${ }^{10}$. Schemas in the vmPFC also support the integration of multiple episode elements and modulate the recall of recent episodic memories $^{13}$, the simulation of future events ${ }^{14}$ and decisionmaking about novel experiences ${ }^{15}$. This integrative function of schemas in the VmPFC, promoting associations between contexts, locations and events, is also critical for synthesizing the emerging affective value of the experienced episode ${ }^{14-16}$. Thus, given that collective schemas require a high level of integration and abstraction to integrate the regularities and recurrent features of collective events, the adaptation of individual memories to a common collective scaffolding may rely on the vmPFC.

The second line of research focuses on the dmPFC, and studies have shown that the functions of this region support the ability to mentalize others' thoughts or social situations ${ }^{17-19}$. The dmPFC is recruited during the processing of situations depicting social interaction $\mathrm{s}^{20}$, is involved during narrative comprehension ${ }^{11}$ and differentiates mental inferences about the group from those about its individual members ${ }^{21}$. This structure has been demonstrated to encode a shared neural structure of narratives across memory patterns of distinct individuals who have a shared experience of the same event ${ }^{22}$. Given its role in representing other people's viewpoints, social situations and story-based narratives, the dmPFC may thus represent persistent social and collective frameworks and provide collective schemas or narratives on which memory can be reconstructed.

We used functional magnetic resonance imaging ( $\mathrm{FMRI}$ ) to test whether participants used collective schemas when remembering pictures seen during a tour of the Caen Memorial Museum (hereafter, the Memorial) in Normandy, France. Memorials play a role in both creating a framework of meaning supporting collective memory and identity, and reactivating a pre-existing collectively shared narrative of history. The purpose of the Memorial tour was therefore not only to activate in our participants a set of knowledge that is consistent with narrative templates that support collective schemas, but also to reshape their understanding and knowledge towards a collective and

\footnotetext{
${ }^{1}$ Neuropsychologie et Imagerie de la Mémoire Humaine U1077, INSERM, EPHE, UNICAEN, Normandie Univ., PSL Research University, CHU de Caen, Cyceron, Caen, France. ${ }^{2}$ IHRIM laboratory UMR 5317, CNRS, ENS de Lyon, Université de Lyon, Lyon, France. ${ }^{3}$ LIMSI, CNRS, Université Paris-Saclay, Orsay, France. ${ }^{4}$ LIUM EA 4023, Le Mans Université, Le Mans, France. ${ }^{5}$ CNRS, HESAM Université, Université de Paris 1 Panthéon Sorbonne, EHESS, UMR8209, European Centre for Sociology and Political Science (CESSP), Paris, France. *e-mail: gagnepain@cyceron.fr
} 
Day 1: encoding phase (memorial tour)

Memorial zones

\begin{tabular}{|c|}
\hline $\begin{array}{l}\text { North African } \\
\text { campaign }\end{array}$ \\
\hline $\begin{array}{l}\text { Invasion } \\
\text { of USSR }\end{array}$ \\
\hline $\begin{array}{c}\text { Atlantic } \\
\text { battle }\end{array}$ \\
\hline $\begin{array}{l}\text { Japanese } \\
\text { expansionism }\end{array}$ \\
\hline Pearl Harbor \\
\hline $\begin{array}{c}\text { Persecution } \\
\text { of Jews }\end{array}$ \\
\hline $\begin{array}{l}\text { Fate of } \\
\text { children }\end{array}$ \\
\hline 3) Gypsies \\
\hline $\begin{array}{l}\text { Japanese } \\
9 \text { violence }\end{array}$ \\
\hline
\end{tabular}
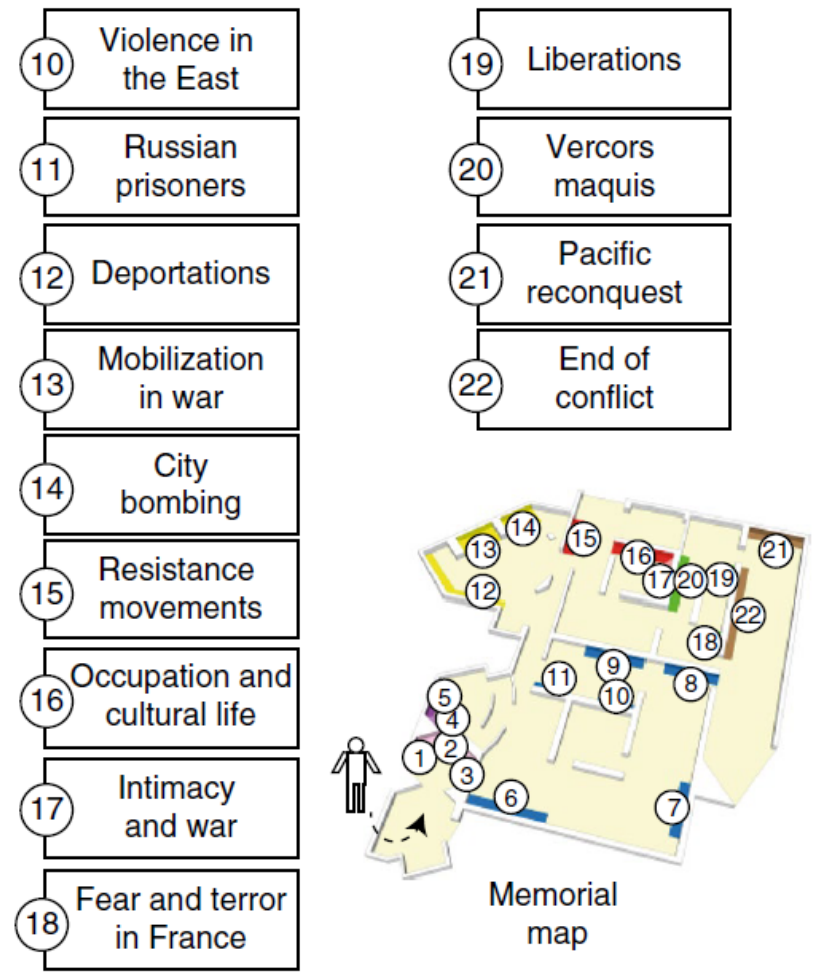

b

Day 2: retrieval phase (fMRI recording)

Recall task

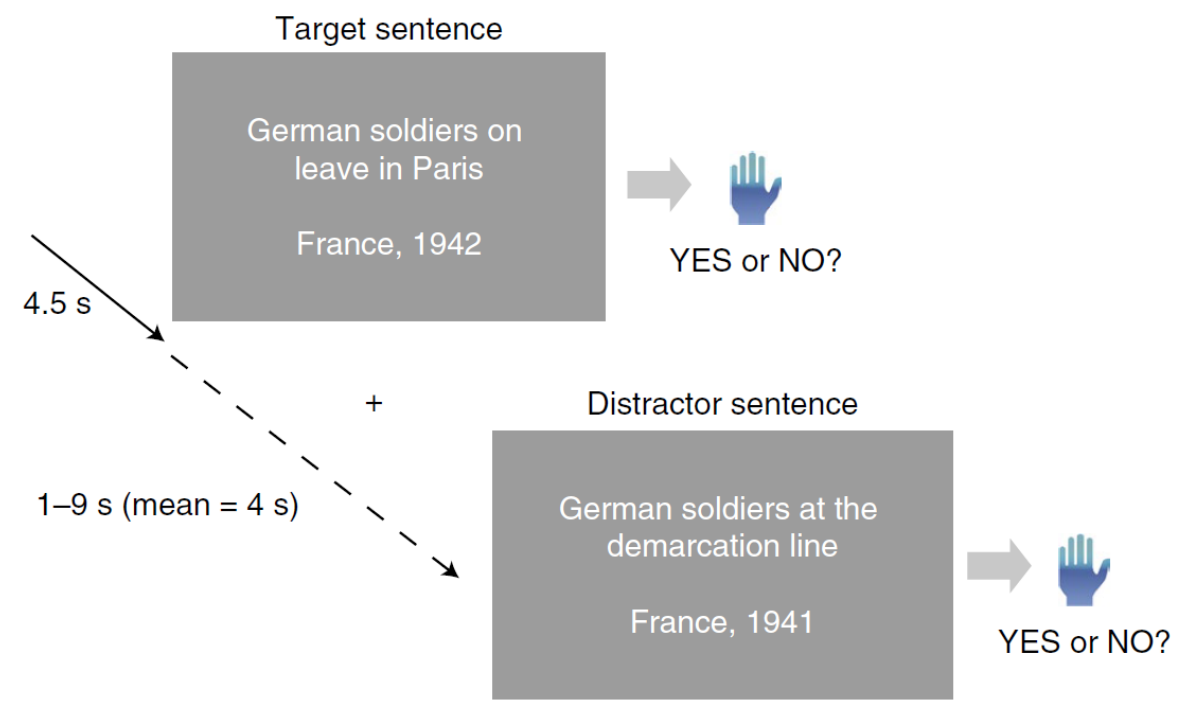

Fig. 1 | Experimental design for recall of individual memories. a, On Day 1, participants toured part of the Caen Memorial Museum. They saw a total of 119 pictures (with captions underneath) in 22 thematic zones (zone numbers in circles). $\mathbf{b}$, The next day, we recorded the participants' brain activity using fMRI while they recalled pictures from short captions comprising either target or distractor sentences. 
shared agreement. A total of 24 healthy participants were led on a tour around the Memorial, where they were exposed to a total of 119 pictures in 22 different zones (Fig. 1a). Each zone was associated with a particular aspect of World War II, and zone order during the tour (six possible routes) was counterbalanced across participants. The next day, we recorded brain activity using fMRI while participants recalled pictures from either short captions describing the pictures they had seen the day before (target sentences) or novel captions (distractor sentences) (Fig. 1b). During this task, participants were asked if they could visualize the picture cued by the short description. Distractor sentences described real events and existing pictures, very similar to those exhibited at the Memorial. Compared with a recognition task of the pictures that may rely on perceptual familiarity, this procedure ensured that participants actively engaged in recalling the exhibited pictures and their associated contents, including the collective body of knowledge, which should, like any other schema, provide a long-term organizational framework facilitating the reconstructive processes engaged during memory recall. Our goal was to demonstrate such topdown influence of collective schemas during recall by assessing the similarity between the memory representations in the MPFC and their collective expressions.

Commemorative events, films, education and numerous memorial sites stress the role of particular historical events and promote their retention in collective memory. These various forms of memories are constantly being produced through the media, which play a central role in expressing and shaping the collective memory of a nation, connecting people through a common narrative across time and space ${ }^{5}$. To capture the organization of collective schemas in the media, we analysed a corpus including all 3,766 news bulletins and reports on World War II broadcast on French national television from 1980 to 2010 (Fig. 2a). This corpus was collected at the National Audiovisual Institute, a repository of all French radio and television archives. For decades, this institute has conserved audiovisual material, with a particular focus on television programmes (mandatory deposit since 1992). Our aim was to analyse this corpus to extract the gist or schema of the semantic relationships between words and to generalize this schema to the Memorial pictures using their captions. Previous approaches to computing the semantic relationships between words include latent semantic analysis (LSA), network analysis, word embedding vectors (that is, Word2vec), and topic modelling based on a latent Dirichlet allocation (LDA). Among these approaches, LSA $^{23,24}$, network analysis ${ }^{25}$ and topic modelling ${ }^{26-28}$ have been applied successfully to provide plausible models of the human semantic memory structure that account well for various memory processes and performances. Here, we choose to focus on topic modelling based on $\operatorname{LDA}^{29,30}$ in which word meanings are represented in terms of a set of probabilistic topics (that is, category or lexical field) that can be used to infer the gist (that is, a distribution over topics) of new documents. In this context, the advantage of LDA is its reliance on a generative mode ${ }^{26}$ derived from words in the collective memory corpus that can be inverted and generalized to the Memorial pictures (using their captions) to describe their topic distribution and their semantic distances.

After speech-to-text conversion and lexical processing, we counted occurrences of 6,240 canonical forms of a set of words (that is, lemmas) across all 3,766 documents to form a Word $x$ Document frequency matrix. We then applied a topic model

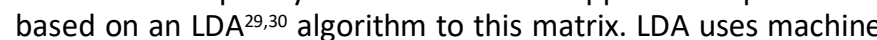
learning to discover latent factors by learning the topics that occur in a collection of documents. In short, the probabilistic topic model estimated by LDA consists of two matrices. The first matrix describes the probability of drawing a particular word when sampling a particular topic. The second matrix describes the probability of finding a particular topic when sampling a particular document (here, television news bulletins and reports). These two matrices, once combined, constitute a model that can generate word frequencies across documents. This generative model is initiated by randomly choosing a topic mixture for a given document (according to a Dirichlet distribution over a fixed set of $K$ topics). This topic mixture spits out words with certain probabilities, and the whole process is refined with Gibbs sampling to establish a set of topics that are likely to have generated the whole corpus. Finally, we fitted this topic model to the Memorial pictures, using their captions (also lemmatized) to estimate their topic probabilities (see Fig. 2a). The distance between the distributions of topic probabilities for each pictures was then encoded in a representational dissimilarity matrix (RDM). This collective RDM reflected the semantic structure of collective memory for Memorial pictures, where the meaning of a given picture was defined by its relative distance from all the other pictures. Given that topic modelling introduces a degree of randomness during parameter estimation, we reiterated the whole process ten times. Each measure of similarity reported below was averaged across these ten repetitions.

\section{Results}

Validation of collective memory measurements. We first ran a series of extra comparisons to validate the model of collective memory computed from the national corpus of news broadcasts. Our goal was to demonstrate the universality and validity of the model of collective memory by showing that it reflects a shared and selective representation of the past, whose core structure is different from a historical perspective that aspires to provide an accurate account of the past. Collective memories are often viewed as shared individual memories or representations resulting from the social means and transmission tools created by the community ${ }^{31}$. These social means serve not only to reshape and harmonize individual representations by guiding the remembering of all the members of the community, but also to regulate the construction of a collective identity by selecting and emphasizing certain elements of the past at the expense of others $^{3-6}$. This fact highlights two fundamental properties of collective memory: (1) collective memory is located both in the social tools used to connect individuals (for example, national news broadcasts) and in individual minds (as a shared form of representation), and (2) the selection and sharing of events for inclusion in the collective memory are determined by their social value and importance to society, leading to a schematization that may contrast with the meaning of historical memory.

We sought to assess how well the collective structure we obtained matched this definition. To this end, we measured the shared pool of knowledge across 54 control individuals unfamiliar with the Memorial, and tested whether this shared representation was a better characterization of the structure of collective memory than a model describing the relatedness of the images from a historical perspective. To capture the shared structure of individual representations, we designed an image arrangement task $\mathrm{k}^{32}$ (Fig. 2b) to capture each individual semantic organization of the Memorial pictures within a common space. During this task, we asked participants to place the images displayed in the Memorial inside circles partitioning the map space, according to their historical proximity (Fig. 2b). These spatial arrangements of historical pictures reflected the semantic organization of each individual, and were encoded in RDMs using the Euclidean distances between the images. We computed the compromise ${ }^{33}$ of these 54 individual RDMs to measure a common representation reflecting a shared schema (that is, a shared RDM; 
Collective memory schemas
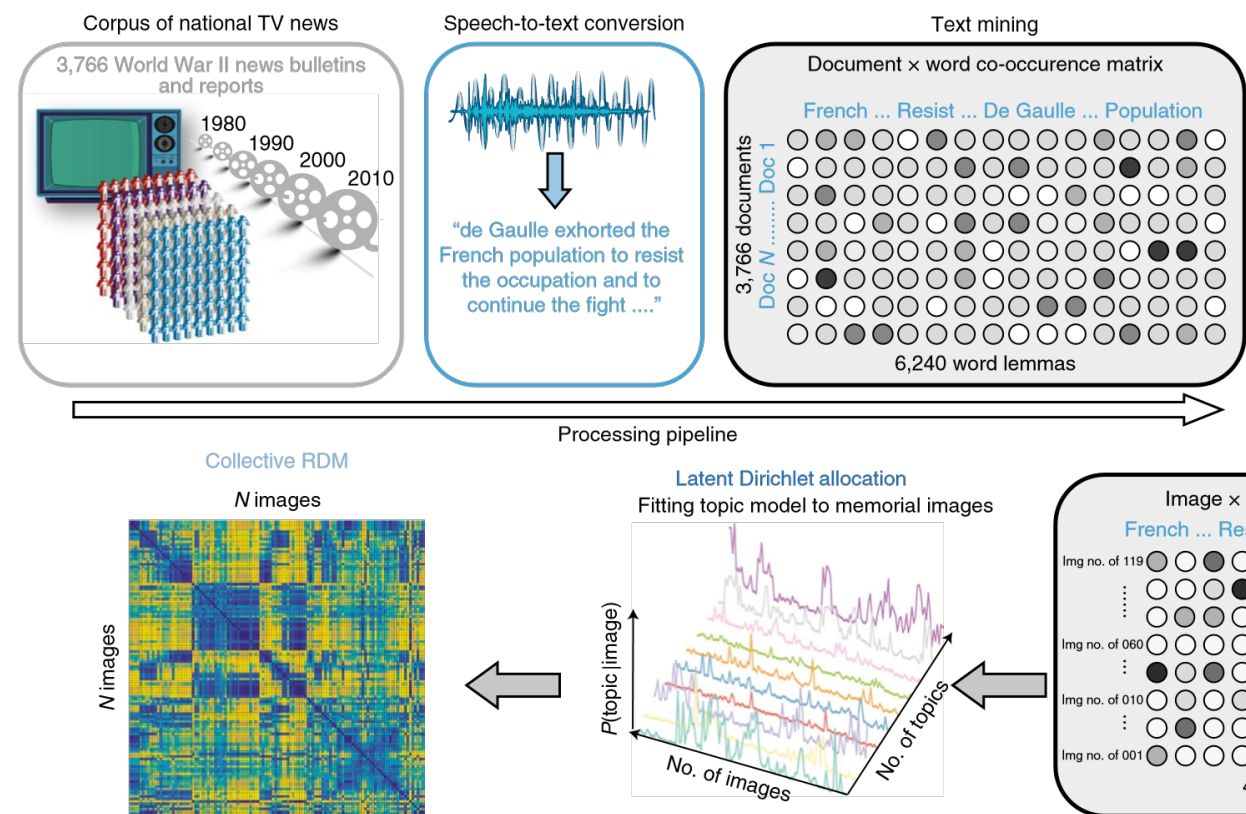

Processing pipeline

Latent Dirichlet allocation
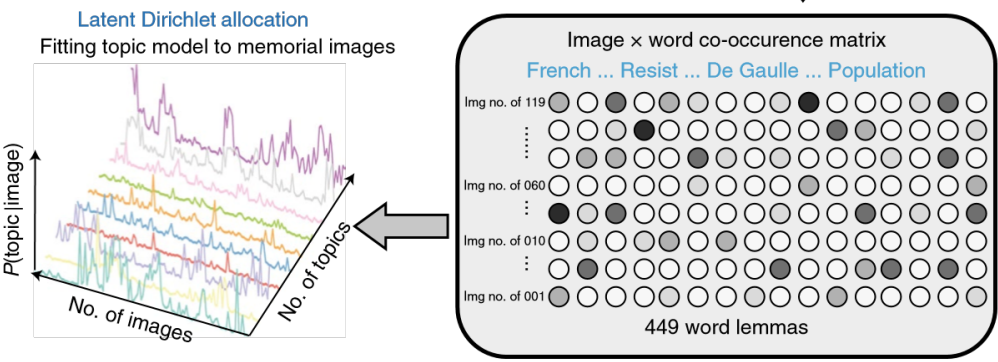

b

Shared memory schemas
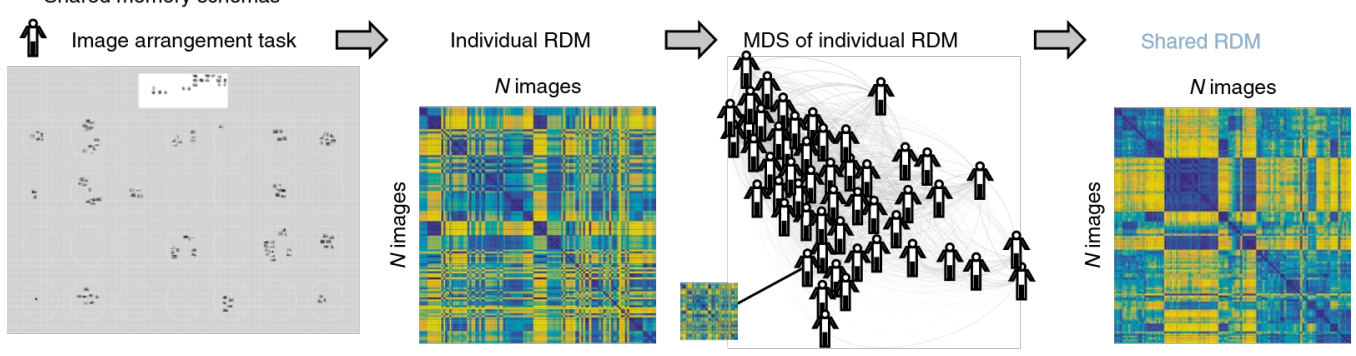

c
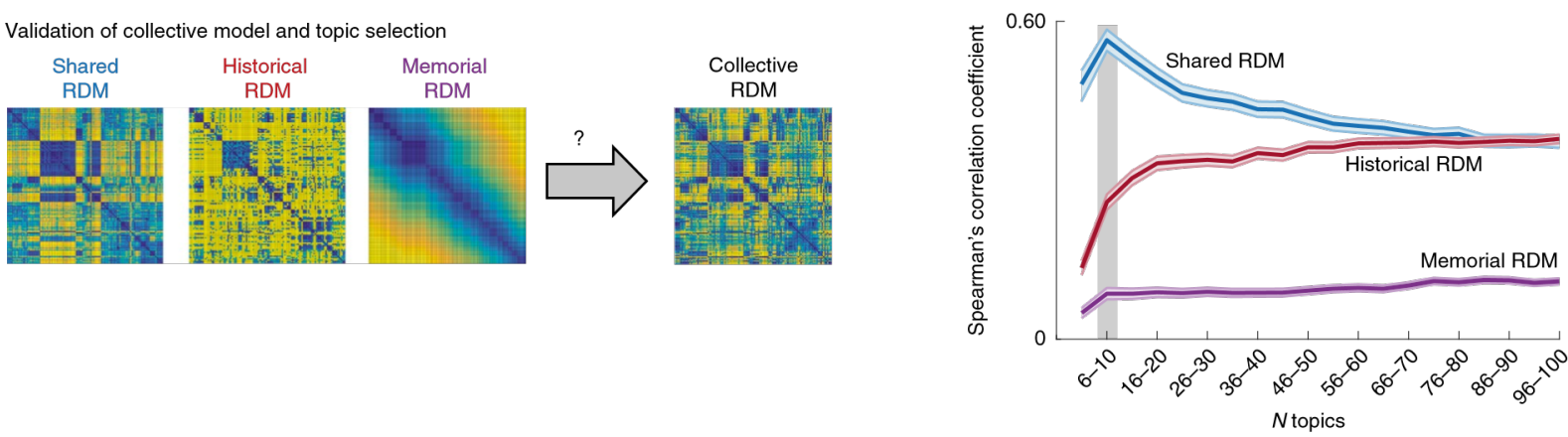

Fig. 2 | Measuring collective, shared and contextual memory. a, Thirty years of television news bulletins and reports were turned into text, lexically processed and analysed using topic modelling. The topic model was then fitted to the Memorial pictures, using their captions to estimate their topic probabilities. The distances between the pictures, based on the distribution of topic probabilities, therefore reflected the semantic properties of the collective memory structure, and could be encoded in an RDM. The whole process of learning and fitting the topic model was repeated by varying the number of topics allowed from 2 to 100 , in increments of 1 . For each topic, topic estimation was also reiterated ten times to account for the small variability in parameter estimation generated by the topic models. $\mathbf{b}$, To demonstrate the universality of the collective memory RDM and independently select the optimum number of topics, we extracted the structure of shared representations across 54 individuals who were unfamiliar with the Memorial, using a task in which they had to arrange images based on their historical proximity. The resulting shared RDM corresponded to the compromise ${ }^{33}$ of all 54 individual RDMs derived from the Euclidean distances of the image arrangement. The relationship between individual RDMs is illustrated by a multidimensional scaling (MDS) plot. c, We computed the similarity of the collective memory RDM, averaging in incremental bins of five topics, to our control model of shared memory (see b), as well as to additional control models encoding the semantic distances of the pictures intended by the Memorial's planners and historians (that is, the Memorial and Historical RDMs, based on the Memorial's zone indices and the historical proximity of the pictures derived from their captions, respectively). The collective memory RDM estimated from the topic model was best predicted by the structure of the shared memory RDM measured across control individuals (right). This similarity between collective and shared memory (error bar reflects $95 \%$ confidence interval, computed by bootstrapping the 54 -individual set) peaked when six to ten topics were included during topic discovery (grey shaded bar). Subsequent analyses of brain and behavioural data involving comparisons with the collective RDM were restricted to this range of topics, which best reflected the content of shared memory across individuals at the expense of historical accuracy. 
Dependencies of model RDMs

Spatial RDM $\bigcirc_{\circ}^{\circ} \bigcirc$ Temporal RDMs

Collective RDMs

Semantic RDMs

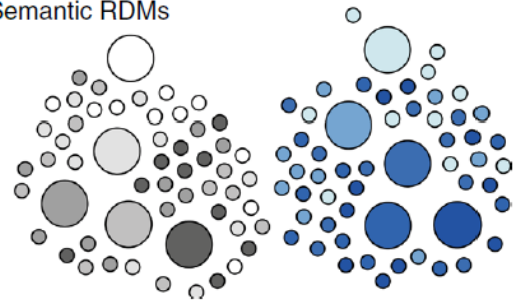

MDS plot b

mPFC ROIs

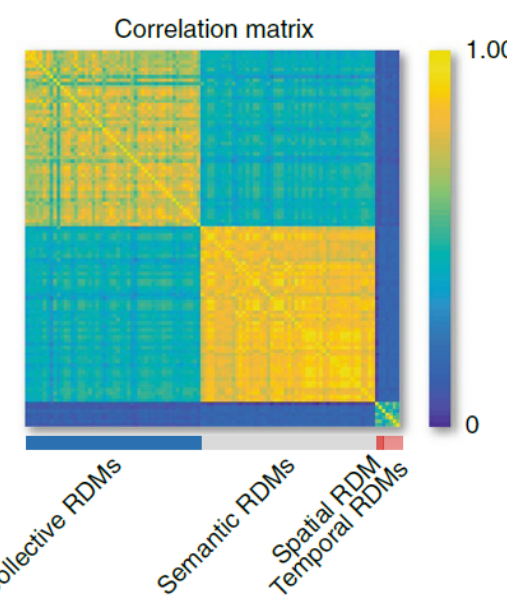

00

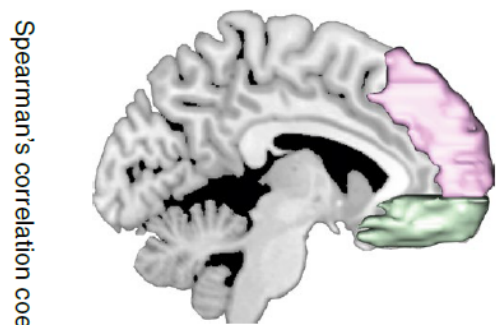

dmPFC

vmPFC

C

RSA results
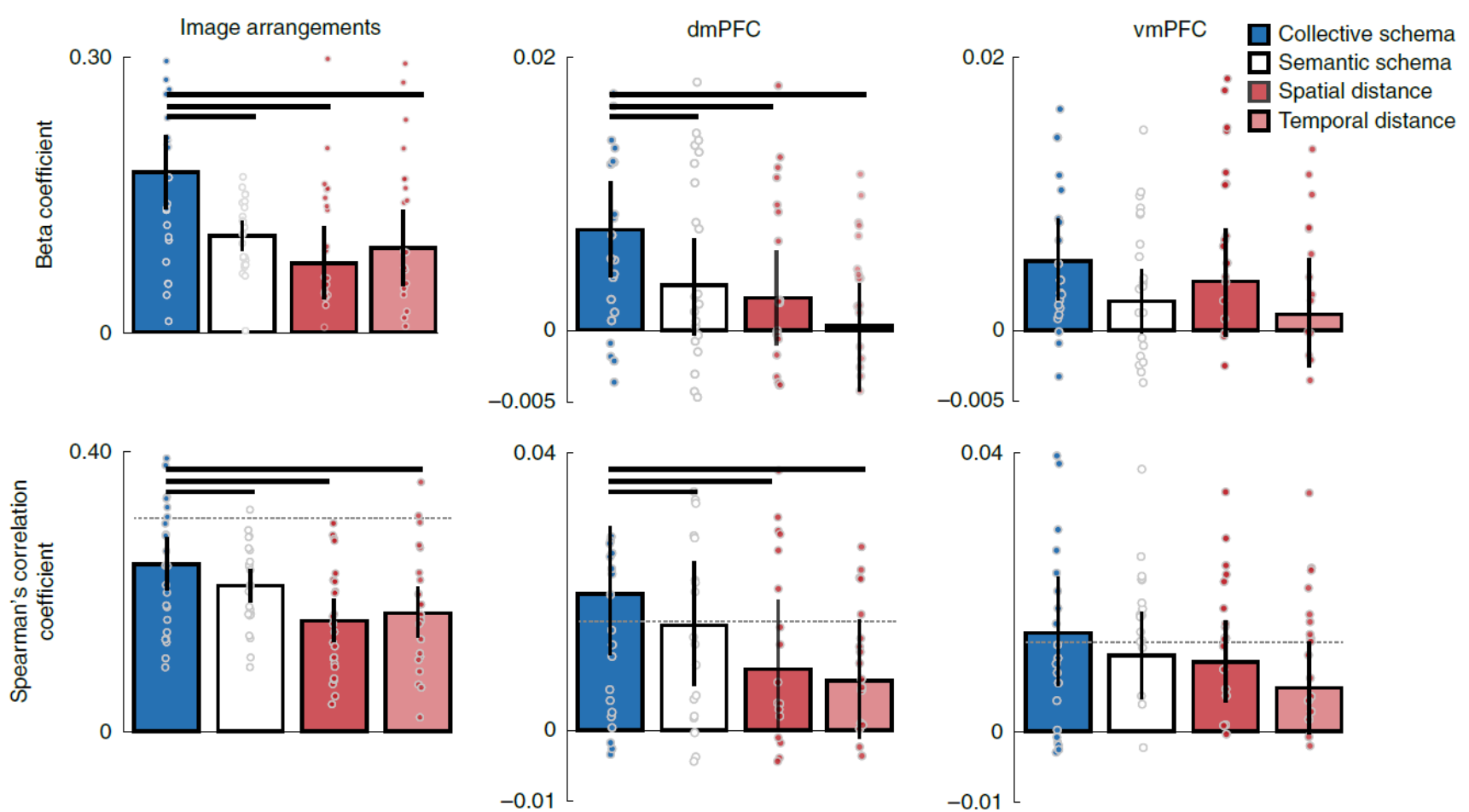

Fig. 3 | Model dependencies and results of the rSA. a, MDS of the RDMs describing the relationships between pictures in terms of their collective (national news bulletins and reports on World War II), semantic (that is, Wikipedia World War II articles), spatial (that is, Memorial layout) and temporal (that is, acquisition order) properties. Collective and semantic RDMs included six to ten selected topics (Fig. 2c) and their ten iterations. Temporal RDMs included the six possible routes around the Memorial. $\mathbf{b}$, The dmPFC and vmPFC regions of interest (ROIs). c, Similarities between the upper triangular portions of image arrangements (left), dmPFC (middle) and vmPFC (right) RDMs, and collective, semantic and contextual model RDMs. Bar graphs display the mean beta coefficients from the regression model (top) and Spearman's correlation coefficients (bottom) across participants ( $N=24$ ). Horizontal lines indicate significant differences at $P<0.05$, false discovery rate (FDR)-corrected for multiple comparisons. Error bars reflect $95 \%$ bootstrapped Cls (and thus indicate significance when they do not overlap with zero). Dashed horizontal lines indicate the noise ceiling (that is, an estimate of the reliability of the neural data; see Methods), which reflects the expected performance of the (unknown) true model given the noise and variability among study participants ${ }^{34}$. Regarding brain imaging data, the collective RDM reaches the noise ceiling, indicating that collective schemas account for a significant amount of the true neural dissimilarity structure. Note that in the context of the current experiment, the small correlation between individuals' brain dissimilarity structures and the expected true model arises from the fact that we have only one measurement of each pattern of memory activity (that is, participants recall the picture only once), making the estimate of the neural dissimilarity noisy. 
Fig. 2c). To capture the historical distance between the pictures, we used the image captions, written by museum curators with indepth historical knowledge of the war, and the relatedness of the events (Historical RDM), as well as the Memorial's own organization, whereby the images were grouped in 22 thematic zones (Memorial RDM). The Historical RDM was derived from a standard vector-space model where each image is represented by a vector of terms including all lemma words occurring in the Memorial captions (but with no knowledge of topic assignment). For each picture, the weights of this vector of terms corresponded to the term frequency (derived from the collective memory corpus) or to zero, depending on the occurrence or absence of the word in the caption describing the picture, respectively. The cosine distances between all possible pairs of pictures' term vectors were used to compute the Historical RDM, while the Memorial RDM was derived from the rank order of the images in the Memorial's main route (Fig. 2c). These two RDMs therefore reflected the semantic proximity of the pictures as intended by the museum's historians and planners.

The collective RDM extracted from the news corpus was very similar to the structure of the shared RDM measured across control individuals (Fig. 2c, right). This similarity between collective and shared memory peaked when six to ten topics were included during topic discovery. Furthermore, the collective RDM was best predicted by the shared RDM, which outperformed both the Historical and Memorial RDMs. In line with the definition of collective memory, these findings therefore suggest that the computed meaning of the images reflected a shared agreement, and the schematic structure of the knowledge shared by control participants unfamiliar with the Memorial was different from the historical proximity of the images defined by the experts. As a result, all subsequent analyses were based on a collective memory RDM computed using six to ten topics.

Representational similarity analysis. Control models. Our aim was to estimate the similarity between the organization of the collective RDM and the organization of individual memory, using representational similarity analysis $\left(\mathrm{RSA}^{34}\right)$. However, the similarity between the patterns of memory activity could be confounded by the expression of episodic memory reflecting the context of acquisition, including temporal and spatial information about the encoded events. This context of acquisition could overlap and share similarities with the structure and organization of collective memory. To differentiate these effects of contextual proximity from the collective schema and to clarify the role of episodic memory for contextual elements, we also tested contextual RDMs describing the organization of pictures in the Memorial (Fig. 3a). These contextual RDMs were derived from the relative spatial distance of the pictures in the Memorial (that is, spatial RDM) and the order in which they were seen during the tour (that is, temporal RDM).

The relationships between the pictures captured by our model of collective memory could also correspond to pre-existing semantic similarities reflecting a form of shared (although not collective) memory for common concepts and the meaning of language. To control for these commonly shared semantic relationships, we used 2,643 French Wikipedia articles related to World War II as a benchmark model for the specific semantic relationships between words related to World War II, and trained a topic model that we then fitted to the Memorial pictures. LDA models of Wikipedia articles about various concepts have been shown to produce an accurate representation of semantic features that effectively predict patterns of brain activity ${ }^{27,28}$. As a global repository of memory, which is not simply stored but is the product of the collaborative recall of many people and has a communicative function, Wikipedia shares several features with the concept of collective memory ${ }^{35}$. There are, however, several critical differences ${ }^{36}$. Collective memory is a selective representation of the past, shaped by schematic narrative templates that contribute to the construction of group identity, often emphasizing some elements while minimizing others ${ }^{3}$. Given its encyclopaedic nature, it is debatable whether Wikipedia fosters this selective representation of the past and the formation and compilation of corresponding memories that have a bearing on group identity. Wikipedia cannot be understood as a consistent medium, in contrast to television news bulletins and reports, which consistently promote symbolic and memorable elements of collective memory without negotiation. For instance, the meaning of the French word 'collaboration' varies between the television news corpus and Wikipedia. In the former, this concept unambiguously refers to the participation of the French government and its politicians and public servants (for example, Bousquet, Leguay, Touvier and Papon) in the deportation and killing of Jews, and the arrest of French Resistance fighters by German Nazis. In World War II-related Wikipedia articles, however, the word 'collaboration' is associated with different semantic contexts that are not necessarily related to French collaboration. By contrast, the semantic domain containing shared meanings in the lexical and conceptual fields related to war is similar across corpuses (for example, 'ship' and 'navy' are related in both corpuses). To control for these shared domainspecific relationships between World War II-related words and concepts, we therefore created semantic RDMs (Fig. 3a) using the 2,643 French Wikipedia articles related to World War II, and applied the same method developed to create the collective RDM.

Behavioural results. To delineate the role of collective memory through the expression of individual memory at the behavioural level, fMRI participants performed the image arrangement task that was used as a proxy for individual schemas (that is, individual RDMs computed using the Euclidean distances between all possible pairs of pictures in this two-dimensional behavioural arrangement). We first sought to assess whether the Memorial tour increased the consistency between individual and collective memory by comparing the similarity of the arrangements of the fMRI and control (that is, unfamiliar with the Memorial) participants with the collective RDM, using Spearman's correlation. One-tailed two-sample $t$-tests confirmed this prediction and revealed the presence of a greater similarity $(t(76)$ $=1.70 ; P=0.047,90 \%$ confidence interval $(\mathrm{Cl})(0.0007,0.088)$, Cohen's $d=0.41$ ) to collective schemas for participants that had explored the Memorial (mean $=0.24$; s.d. $=0.095$ ) compared with the control group (mean $=0.196 ;$ s.d. $=0.12$ ). These results suggest that experiencing a visit at the Memorial reshapes and harmonizes individual knowledge towards a shared configuration, solidifying collective schemas. However, this difference could also be driven by the fact that control participants were less familiar with the pictures when they had to arrange them, perhaps impacting subtle arrangements they could make within the set of pictures.

We next sought to assess which model (collective, semantic or contextual) best explained the expression of individual memory at the behavioural level. The upper triangular portions of these model RDMs were entered in a regression model as predictors of the upper triangular portions of the individual RDMs (see Chikazoe et al. ${ }^{37}$ and Bracci et al. ${ }^{38}$ for a similar approach). Both regressors and data were initially rank-transformed to test for nonlinear monotonic relationships. Given that collective, semantic (that is, Wikipedia) and contextual (that is, spatial and temporal) model RDMs were not orthogonal and overlapped to some extent (see Fig. 3a), we used regression models to identify 
the unique variance attributable to each of the model predictors. We report the results of these regression models as well as the results of standard Spearman's correlation coefficients calculated in isolation, for the sake of completeness, in Fig. 3c and Supplementary Table 1. Group-level inference on RDM model relatedness and differences were estimated using nonparametric random-effects statistics by bootstrapping the participant set (see Methods for details). At the behavioural level, individual RDMs were significantly related to the collective RDM (bootstrapped $P_{\mathrm{FDR}}<0.0001$, bootstrapped $95 \% \mathrm{Cl}(0.1373$, 0.2109 ), Cohen's $d=1.82$ ), an effect that was critically stronger than the similarity with semantic (bootstrapped $P_{\text {FDR }}<0.0001$, $95 \% \mathrm{Cl}(0.0321,0.1066)$, Cohen's $d=0.73)$, spatial (bootstrapped $P_{\mathrm{FDR}}<0.001$, bootstrapped $95 \% \mathrm{Cl}(0.0421,0.1562)$, Cohen's $d=$ 0.67 ) and temporal (bootstrapped $P_{\mathrm{FDR}}=0.002$, bootstrapped $95 \% \mathrm{Cl}(0.0277,0.1348)$, Cohen's $d=0.60)$ RDMs (Fig. 3c, left). These findings suggest that knowledge is organized around collective schemas in individual brains.

Brain imaging results. To further test this hypothesis, we extracted the pattern of activity across voxels of the dmPFC and vmPFC (see Fig. 3b) for each recalled memory of the pictures, and we characterized the RDM by computing the correlation distances (1 - Pearson correlation coefficient across voxels) between all possible pairs of memory patterns. Confirming our main hypothesis that the mPFC codes collective schemas, both dmPFC (bootstrapped $P_{\mathrm{FDR}}<0.0001$, bootstrapped $95 \% \mathrm{Cl}$ $(0.0042,0.0108)$, Cohen's $d=0.89$ ) and vmPFC (bootstrapped $P_{\text {FDR }}$ $<0.0001$, bootstrapped 95\% Cl $(0.0019,0.0083)$, Cohen's $d=$ $0.67)$ RDMs were significantly related to the collective RDM (Fig. $3 c$, middle and right, respectively). Patterns of activity in the dmPFC were moderately related to semantic (bootstrapped $P_{\mathrm{FDR}}$ $=0.0514$, bootstrapped $95 \% \mathrm{Cl}(-0.0003,0.0067)$, Cohen's $d=$ 0.39 ) and spatial (bootstrapped $P_{\mathrm{FDR}}=0.0927$, bootstrapped $95 \%$ $\mathrm{Cl}(-0.0008,0.0058)$, Cohen's $d=0.28)$ RDMs, but not to temporal RDMs (bootstrapped $P_{\mathrm{FDR}}=0.4430$, bootstrapped $95 \% \mathrm{Cl}$ $(-0.0035,0.0043)$, Cohen's $d=0.03)$. The similarity of the dmPFC representational content to the collective RDM was stronger than the similarity to the semantic (bootstrapped $P_{\mathrm{FDR}}=0.046$, bootstrapped $95 \% \mathrm{Cl}(0.0001,0.0081)$, Cohen's $d=0.40)$, spatial (bootstrapped $P_{\mathrm{FDR}}=0.026$, bootstrapped $95 \% \mathrm{Cl}(0.0010$, $0.0094)$, Cohen's $d=0.46$ ) and temporal (bootstrapped $P_{\mathrm{FDR}}=$ 0.026, bootstrapped 95\% Cl $(0.0013,0.0128)$, Cohen's $d=0.49)$ RDMs. This result indicates that the representation of the collective schema in the dmPFC during memory recall could not be accounted for by the reactivation of either semantic information or recent contextual memories. A searchlight analysis across the whole brain confirmed the involvement of the mPFC in representing the collective schema, although the collective schema was also found to influence activity in the dorsolateral PFC, an effect previously linked to schema incongruency ${ }^{39}$ (Extended Data Fig. 1). Patterns of activity in the vmPFC were also moderately related to semantic (bootstrapped $P_{\text {FDR }}=0.0576$, bootstrapped $95 \% \mathrm{Cl}(-0.0003,0.0046)$, Cohen's $d$ $=0.34$ ) and spatial (bootstrapped $P_{\mathrm{FDR}}=0.0576$, bootstrapped $95 \% \mathrm{Cl}(-0.0005,0.0074)$, Cohen's $d=0.36)$ RDMs (although these two models were significant when isolated correlations were considered; Fig. 3c, bottom, and Supplementary Table 1). There was no statistically significant evidence that the temporal RDMs explained patterns of activity in the vmPFC (bootstrapped $P_{\mathrm{FDR}}=0.2964$, bootstrapped $95 \% \mathrm{Cl}(-0.0027,0.0054)$, Cohen's $d$ $=0.11$ ) (Fig. 3c, right). However, no statistically significant differences were observed between the collective RDM and the semantic, spatial and temporal RDMs in the vmPFC $\left(P_{\text {FDR }}>0.197\right.$; see Supplementary Table 1). These findings therefore suggest that the $\mathrm{vmPFC}$ recalled elements whose representations coincided with the collective meaning, World War II semantic concepts and the spatial layout of the Memorial images.

Memory patterns in the dmPFC and vmPFC showed a consistent relationship with collective memory. For the dmPFC patterns, comparisons with the control semantic model based on Wikipedia World War II articles suggested that this effect was not confounded by pre-existing semantic memory for common concepts and the meaning of language, and comparisons with models accounting for contextual elements of the Memorial tour suggested that it was not confounded by episodic memory for contextual details. Such distinctions were not, however, observed in the vmPFC. These findings thus suggest that the dmPFC encodes a common mental model reflecting the structure of collective memory computed from the topic modelling of the national corpus of news broadcasts. We performed an additional analysis to confirm that this relationship was not related to a trivial effect of word historical proximity and that topic models based on LDA better explain the underlying neural representations of the picture memory traces. This analysis entailed a comparison of the collective RDM with the standard vector space model encoding the historical proximity of the words (that is, the Historical RDM; Fig. 2c) but with no knowledge of collective topic assignment. The similarity of the representational content to the collective RDM was stronger than the similarity to the Historical RDM for both the dmPFC (bootstrapped $P=0.033$, bootstrapped 95\% Cl $(0.001,0.015)$, Cohen's $d=0.43$ ) and the vmPFC (bootstrapped $P=0.028$, bootstrapped $95 \% \mathrm{Cl}(0.001,0.014)$, Cohen's $d=0.44)$. This result confirmed that our initial observation does not reflect a trivial effect of word proximity in the captions.

Collective schemas are integrated in the course of a lifetime. In theory, we would expect exposure to the relevant World War II media to increase the alignment of individual schemas with the collective schema, which would then be apparent in the pattern of neural activity. As such, we might expect to see individual agerelated differences, such that more exposure to the relevant World War II media would increase alignment with the collective schema. Older individuals should have more exposure to the relevant World War II media and presumably exhibit a closer alignment with the collective schema. In line with this idea, we found a robust correlation ${ }^{40}$ between age and neural similarity with the collective schema in the dmPFC (coefficient of correlation $(r)=0.3559, P=0.044$, bootstrapped $95 \% \mathrm{Cl}(0.0053$, 0.6535); no bivariate outlier detected) but not in the vmPFC ( $r=$ $0.0805, P=0.35$, bootstrapped $95 \% \mathrm{Cl}(-0.2944,0.4942)$; one bivariate outlier detected). However, no such statistically significant correlation was observed for the Wikipedia RDM in either the dmPFC ( $r=0.1117, P=0.30$, bootstrapped $95 \% \mathrm{Cl}$ $(-0.3325,0.4933)$; no bivariate outlier detected) or the vmPFC $(r$ $=0.2237, P=0.14$, bootstrapped $95 \% \mathrm{Cl}(-0.3029,0.6186)$; no bivariate outlier detected). These findings further support the idea that collective schemas are represented by the dmPFC and are constantly enriched by experiences, through repeated exposure and extraction of commonalities.

We then transformed the individual (derived from the imagearrangement task) and collective RDMs into a binary matrix to further disentangle the contributions of collective and individual schemas to the pattern of neural activity observed during memory recall (Fig. 4, top right). This transformation allowed us to distinguish between the common and unique contributions of collective and individual schemas to the pattern of neural dissimilarity. For each arrangement in the image arrangement task, we extracted a binary adjacency matrix by considering the images that were placed in the same circles (see Methods and Fig. 2b). These circles could be viewed as proxies for individual semantic categories, and binary matrices were created 
accordingly ( 1 indicating pictures connected by the same category and 0 indicating disconnected pictures). We then applied the same rationale to the distribution of topic probability, to obtain a collective adjacency binary matrix, but this time considering that two pictures belonged to the same category if they shared the same maximum topic assignment. We then broke these individual and collective adjacency binary matrices down into three distinct matrices reflecting connections between pictures that were (1) unique to the individual schema (individual connections), (2) unique to the collective schema (collective connections) and (3) common to the individual and collective schemas (common connections; see Fig. 4, bottom left). We then transformed these matrices into distance matrices and computed the similarities with the brain RDMs (Fig. 4, bottom right). Although on average, similar numbers of connections were specific to the individual schema or common to the individual and collective schemas, neural similarities in the dmPFC (bootstrapped $P_{\mathrm{FDR}}<0.0001$, bootstrapped $95 \% \mathrm{Cl}(0.0064$, 0.0171 ), Cohen's $d=0.83$ ) and vmPFC (bootstrapped $P_{\mathrm{FDR}}=$ 0.0012 , bootstrapped $95 \% \mathrm{Cl}(0.0024,0.0099)$, Cohen's $d=0.63)$ were preferentially related to common connections. Although individual (bootstrapped $P_{\mathrm{FDR}}=0.0016$, bootstrapped $95 \% \mathrm{Cl}$ $(0.0023,0.0118)$, Cohen's $d=0.58)$ and collective (bootstrapped $P_{\mathrm{FDR}}=0.0016$, bootstrapped $95 \% \mathrm{Cl}=(0.0023,0.0118)$, Cohen's $d$ $=0.59$ ) connections were both significantly represented in the dmPFC, the dmPFC's representational contents were more similar to the common connections than to either purely individual (bootstrapped $P_{\mathrm{FDR}}=0.021$, bootstrapped $95 \% \mathrm{Cl}$ (0.0006, 0.0083), Cohen's $d=0.46$ ) or purely collective (bootstrapped $P_{\mathrm{FDR}}=0.0096$, bootstrapped $95 \% \mathrm{Cl}$ (0.0024, $0.0130)$, Cohen's $d=0.55)$ connections. These distinctions between common connections on the one hand, and individual (bootstrapped $P_{\mathrm{FDR}}=0.002$, bootstrapped $95 \% \mathrm{Cl}(0.0017$, 0.0071 ), Cohen's $d=0.62$ ) and collective (bootstrapped $P_{\mathrm{FDR}}=$ 0.037 , bootstrapped $95 \% \mathrm{Cl}(0.0003,0.0075)$, Cohen's $d=0.42)$ connections on the other, were also found in the vmPFC, which also significantly represented collective connections ( $P_{\mathrm{FDR}}=$ $0.0303,95 \% \mathrm{Cl}(0.0001,0.0041)$, Cohen's $d=0.40)$. We found no statistically significant evidence that the vmPFC represented individual connections (bootstrapped $P_{\mathrm{FDR}}=0.1585$, bootstrapped $95 \% \mathrm{Cl}(-0.0015,0.0049)$, Cohen's $d=0.20)$. These findings therefore suggest that remembering is driven by our individual understanding of representations that reflect our experience of social and collective constructs. Individuals' remembering and understanding of the past is linked to their consciousness and knowledge of collective and social group memory.

\section{Discussion}

Previous brain imaging studies have focused entirely on experimentally induced schemas isolated from any collective context. These studies have critically stressed the role of the mPFC in retrieving ${ }^{39}$ or deriving ${ }^{41}$ schematic knowledge from recent episodes. Here, we found that patterns of activity in the dmPFC contained information about the collective meaning of recalled pictures, even after controlling for the influence of other potential confound variables, such as memory for contextual episodic elements (that is, spatial and temporal location) and domain-specific language meanings derived from a collaborative encyclopaedia (that is, Wikipedia). These differences were statistically significant whether the similarities between neural representations and competing models were tested in isolation or in a multiple regression model, suggesting that elements unique to collective memory are represented in the dmPFC. Collective remembering ${ }^{3}$ through social tools shapes memory ${ }^{7,8}$, and groups can also distort individual memories ${ }^{42}$. This memory convergence across individuals emerges out of social interactions in a bottom-up fashion and bolsters group cohesion and identity. Our data demonstrate that, in addition to these social interactions, collectively meaningful information in the media, and therefore stored both outside and inside individuals, constitutes a common pre-existing scaffolding for building individual memories in the dmPFC.

The dmPFC plays a central role in the neural network of narrative comprehension and gist extraction ${ }^{11,22}$. Narratives are key to the elaboration and formation of collective representations, and share some similarities with the concept of memory schema ${ }^{10}$. The dmPFC is also often associated with the ability to infer other people's mental states ${ }^{11,21}$, or theory of mind. Our findings for the dmPFC may therefore reflect the ability of this region to comprehend a collective viewpoint or meaning that is relevant to a given society, facilitating the sharing of a common experience with others ${ }^{43}$. Moreover, the ability to model other people's viewpoints is elicited even when it is currently irrelevant to the task goal ${ }^{44}$. This is reminiscent of our experimental setting, which required individuals to recall their previous personal experience without appealing to a collective viewpoint. Our data therefore suggest not only that representations in the dmPFC may contain information about the collective perspective or meaning of memory content, but also that this reinstatement may occur spontaneously and automatically during memory recall. Our analyses further suggest that individuals align their cognitive knowledge map with the structure of collective memory, and preferentially reactivate these shared representations during memory recall, instead of the specific elements of their individual knowledge. This collective memory that exists outside and lives beyond individuals, yet somehow connects them, could constitute a common model supporting interpersonal communication ${ }^{45}$, thus underscoring the social function of memory 1,46 .

The VmPFC indistinguishably reflected information about collective, semantic and spatial memory when correlations between neural representations and models were tested in isolation, suggesting the existence of a common mapping between these various levels of representations during memory reactivation in this region. This result is in line with recent findings highlighting the primary role of the vmPFC in integrating abstract knowledge in a spatially coherent way using grid cells ${ }^{47}$, and suggesting an integrative function of schemas in the vmPFC to promote associations between contexts, locations and events ${ }^{13-15}$. Our findings therefore suggest that we remember in the vmPFC by transporting ourselves into a persistent conceptual and spatial framework.

Although our findings suggest that the reinstatement of a shared collective schema in the dmPFC during memory recall did not depend on episodic details of the encoded event, it is worth pointing out that this reactivation occurred after just one night of consolidation following the Memorial tour. The Memorial setting may therefore have favoured the rapid formation of a collective schema (as suggested by the greater similarity between individual and collective RDMs following the Memorial tour compared with control participants unfamiliar with the Memorial). Indeed, memorials probably contribute to the establishment of collective schemas in individuals as much as they reflect pre-existing ones. Accordingly, the current data do not allow us to say whether the structure of collective knowledge was present in participants' brains before the Memorial tour. Further studies will be required 

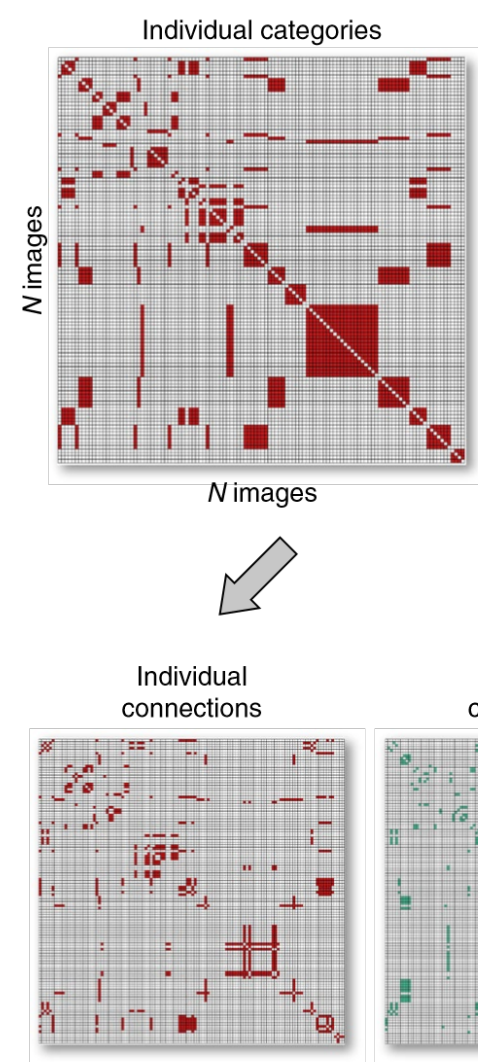
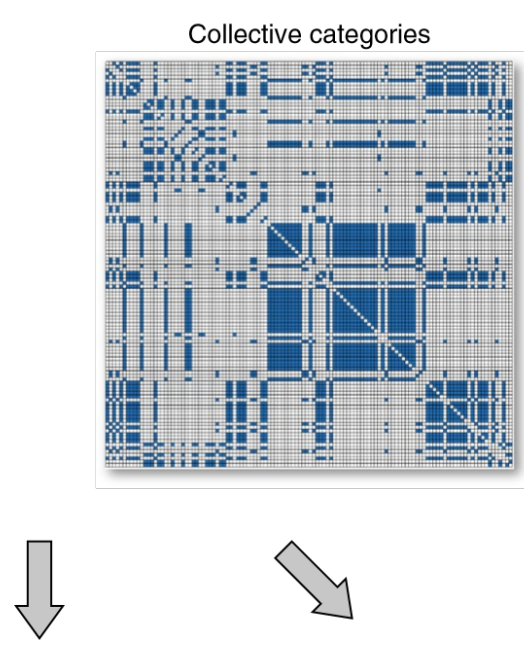

Common connections

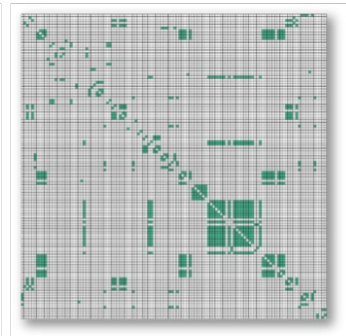

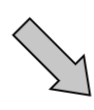

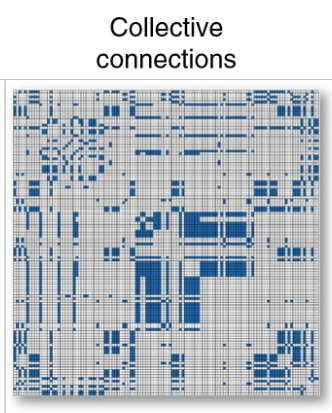

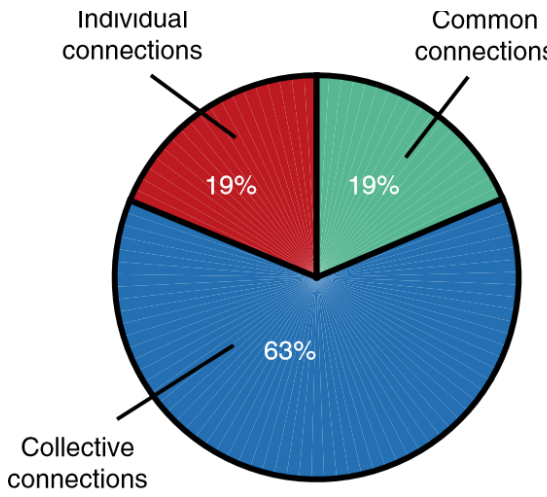

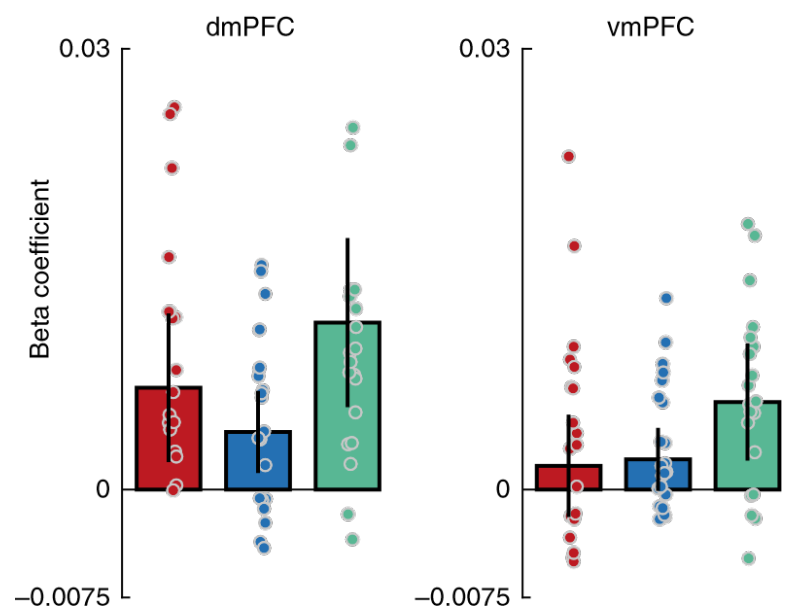

Fig. 4 | Disentangling the contributions of individual and collective schemas. The top left panels show the individual (that is, Participant 8's performance on the image arrangement task) and collective (Topic 8 and Iteration 1 in this example) binarized matrices. Colours indicate that the two pictures belonged to the same category, according to individual (red) and collective (blue) schemas. The bottom left panels display connections (in red) specific to Participant 8 that were not found in the collective schema, common connections (in green) between the individual and collective levels, and connections (in blue) specific to the collective schema and not found in Participant 8. The top right panel shows the relative proportions of individual, collective and common connections, averaged across participants. The bottom right panels show the similarity between the upper triangular portions of the brain RDMs and the individual, collective and common connections models $(N=24)$. Horizontal lines indicate significant differences at $P<0.05$, FDR-corrected for multiple comparisons. Error bars reflect $95 \%$ bootstrapped $\mathrm{Cls}$ (and thus indicate significance when they do not overlap with zero).

to assess whether collective knowledge also helps interpret the flow of ongoing information during memory encoding, and whether its expression is necessarily embedded in the expression and retrieval of episodic memory traces.

Humans maintain group consistency and identity across space and time (generations) using collective memory, which is transmitted by specific cultural tools and means. The current findings do not allow us to state whether the collective schemas identified here are representative of this fundamental cultural specificity and property of collective memory. Even so, our findings highlight the role of the media in promoting the formation of a body of knowledge in the MPFC consistent across individuals, fostering the formation of new related memories in a shape and expression that are harmonized and shared across individuals. Wikipedia, however, despite its collaborative nature, does not seem to foster a collective representation of the past that harmonizes neural representations at individual levels and promotes the compilation of corresponding memories. Although this difference might suggest that Wikipedia cannot be understood as a consistent medium (in contrast to television news bulletins and reports, which consistently promote symbolic and memorable elements of collective memory without negotiation), functional properties of Wikipedia may also explain our findings. For instance, it has been recently shown that a small number of Wikipedia editors have a disproportionately large influence on the formation of collaborative knowledge ${ }^{48}$. The citation system of Wikipedia articles also reduces the content overlap between articles, resulting in weaker and less stable semantic relationships between undisclosed but still related terms in hyperlinks.

The ability or inability to integrate and fit one's personal history or memory into a collective framework imposed by one's cultural environment may play a fundamental role in human wellbeing and deserves to receive greater attention in future studies. These future challenges for the neuroscience of memory can be met only by adopting a transdisciplinary perspective that promotes interactions between psychological, social and mathematical models of largescale data to construct an integrative account of human memory ${ }^{49}$. The present study shows how one such model of memory systems could be constructed, drawing on the interactions between the neurobiological and collective levels.

\section{Methods}

MRI participants. Twenty-four right-handed native French speakers between 22 and 39 years old (mean $=28.6$ years; s.d. $=4.4$ ) were paid to participate (11 males, 13 females). They had no reported history of neurological, medical, visual or memory disorders. The study was approved by the regional research ethics committee 
(Comité de Protection des Personnes Nord-Ouest III, sponsor ID: C13-46, RCB ID: 2014A00126-41, clinicaltrial.gov registration number: NCT02172677). Two additional participants were also initially recruited for design setting and adjustment (but not analysed). One participant was replaced without further analyses due to important MRI artefacts preventing image analysis. All participants gave written consent before participating. Participants were asked not to consume psychostimulants, drugs or alcohol before or during the experimental period. No statistical methods were used to predetermine the sample size, but the sample size used in this study $(N=24)$ is comparable with that of previous $\mathrm{fMRI}$ studies using RSA.

Materials and Memorial exploration procedure. The stimuli were 119 pictures selected from the area World War, Total War at the Caen Memorial Museum. Each display was photographed at the Memorial using a professional digital camera and professional lighting. These pictures were then adjusted for contrast and lighting, and the external contour was cropped, using image processing software.

Each participant explored the Memorial at the end of the afternoon, just before the Memorial door closes. This ensured that other visitors would not disrupt the participants' tours. Participants were first given general instructions about the experiment and were each equipped with a mobile eye-tracking system (Applied Science Laboratory) consisting of glasses and a small storage device connected to the glasses and carried as a backpack. These glasses were mounted with a small camera filming the participant's visual exploration, which was recorded and broadcast on the experimenter's laptop. Although we do not present these eyetracking data, which go beyond the scope of the current paper, we could thus track participants' exploration without following them, and thus ensure that they were complying with the following instructions.

Participants were told to explore a restricted space of the Memorial and were given a map describing the spatial layout (see Fig. 1a). This space comprised a total of 119 pictures, each with a caption underneath, and was organized according to 22 thematic zones (zone numbers are indicated in the circles in Fig. 1a). Two other zones were included at the very beginning and at the very end of the tour for recency and primacy effects. These filler zones were always the same, and their pictures were not included in the subsequent experimental protocol (and were only used for training and familiarization with the subsequent recall task). These 22 zones were grouped according to 6 main sectors (illustrated with the 6 main colours on the Memorial map in Fig. 1a). The exploration order of these main sectors was counterbalanced across participants. The exploration order of the zones within each sector was also randomized according to 6 different exploration lists (4 participants were assigned to each of these 6 lists). Each zone started with an introduction board describing the content of the zone that participants were instructed to read first before exploring the zone. Participants then had to explore each picture composing the zone. They did so by first reading the caption underneath, and then were free to explore the content of the picture as long as they wished before moving on to the next picture until they completed their tour, whose average duration was $76 \mathrm{~min}(\mathrm{~s} . \mathrm{d} .=13.8)$. Note that during this Memorial tour, participants were unaware that their memories would be tested the next day.

Recall task. The next day, participants performed the recall task, which was divided into three fMRI sessions, each about $10 \mathrm{~min}$ in length. Each session presented short sentences corresponding to short excerpts describing real World War II pictures that the participants had explored (that is, target sentences; average word count $=7.8$, s.d. $=2.4$ ) or had not explored (that is, distractor sentences; average word count $=7.7$, s.d. $=2.1$ ) the day before. In total, 119 target sentences and 63 distractor sentences were presented randomly to the participants. Historical events associated with the distractor sentences were selected to match the pictures displayed at the Memorial in terms of both content and relative proportion of pictures per zone. The country in which the event took place was also displayed underneath each sentence, as was the year. Participants were aware of the proximity between distractor and target sentences and were therefore strongly encouraged to rely on their ability to fully recollect and visualize the associated pictures to perform this task. After the onset of each cueing sentence, participants reported whether or not they could recall the associated picture by pressing yes with their right index finger or no with their right middle finger. The sentences appeared for $4.5 \mathrm{~s}$ centred on a grey background. The trials were presented in a stochastic fashion according to a Poisson distribution $(\lambda=4)$ with a $4.2 \mathrm{~s}$ average interstimulus interval (range $=1-10 \mathrm{~s}$ ) with $25 \%$ additional null events and were separated by a fixation cross. Accuracies and performances on the recall task are described in Supplementary Table 2.

Image arrangement task. Outside the scanner, participants performed an image arrangement task, used as a proxy for individual schemas, on the 119 Memorial pictures. The images had to be positioned within 1 to 28 circles according to their historical proximity. This image arrangement task was written in Javascript embedded into HTML code for internet navigation, thus offering great flexibility in the task execution: participants could zoom in or out with a moving background similar to Google Maps, they could enlarge a picture by clicking on it (with the Memorial caption underneath), the Memorial captions appeared on mouseover, and participants could select and move multiple pictures at once. Pictures were initially placed in a large square above the circles. Participants were instructed to review each picture and to place them in the circles below as they went through each of them. They were told to group in the same circle any pictures they felt described close or similar historical events. If they felt the pictures described disconnected events, they were instructed to place them in different circles. Participants were free to use as many circles as they wanted, from a single circle to all circles available on the map. The instructions emphasized that there was absolutely no right number of circles to be used, and that they were free to proceed as they wished. Participants were also instructed to pay attention to the distances between circles and their relative positions. The more they judged that the pictures were linked to connected or disconnected events, the closer or further away their relative positions across circles should be. Finally, when the main arrangement was completed for all pictures, participants had to readjust the positions of the pictures within each circle. The Euclidean distances between the image positions then reflected the semantic organization of a given individual and could be encoded in an RDM.

MRI acquisition parameters. MRI data were acquired on a $3 \mathrm{~T}$ Achieva scanner (Philips) at the brain imaging Cyceron centre in Caen. All participants first underwent high-resolution T1-weighted anatomical volume imaging using a threedimensional (3D) fast field echo (FFE) sequence (3D-T1-FFE sagittal; TR $=20 \mathrm{~ms}$, TE $=4.6 \mathrm{~ms}$, flip angle $=10^{\circ}$, SENSE factor $=2,180$ slices, slice thickness $=1 \mathrm{~mm}$, no gap, field of view $=256 \times 256 \times 180 \mathrm{~mm}^{3}$, matrix $=256 \times 130 \times 180$ ). This acquisition was followed by the functional sessions, which were acquired using an ascending T2-star EPI sequence (MS-T2-star-FFEEPI axial; TR $=2050 \mathrm{~ms}, \mathrm{TE}=30 \mathrm{~ms}$, flip angle $=78^{\circ}, 32$ slices, slice thickness $=3 \mathrm{~mm}, 0.75 \mathrm{~mm}$ gap, matrix $=64 \times 63 \times 32$, field of view $=192 \times 192 \times 119 \mathrm{~mm}^{3}, 310$ volumes per run).

MRI preprocessing. Data were analysed using Statistical Parametric Mapping software (SPM12, Wellcome Department of Imaging Neuroscience). During preprocessing, images were first spatially realigned to correct for motion and were then corrected for slice acquisition temporal delay. After coregistration with the T1 structural image, functional images were then normalized using the parameters derived from the nonlinear normalization of individual 
grey-matter T1 images to the T1 template of the Montreal Neurological Institute. Note, however, that unwarped and unsmoothed images were used for the RSA. Image normalization was nonetheless needed to compute the forward deformation field and its inversion, to normalize searchlight images or wrap back mPFC ROIs to native space (see below), respectively. The use of unsmoothed images is important for RSA as it preserves the finegrained spatial pattern that characterizes the representational geometry of a region.

First-level analysis. The preprocessed time series, corresponding to native space images (that is, non-warped and unsmoothed images), were then high-pass filtered to $1 / 128 \mathrm{~Hz}$ in each voxel. Regressors in a general linear model (GLM) for each voxel were created by convolving a delta function (modelled as $4.5 \mathrm{~s}$ short-epoch) at the stimulus onset for each condition of interest with a canonical haemodynamic response function (HRF). A least-squares separate approach was used ${ }^{50,51}$, which consisted of estimating a separate GLM for each trial. In each GLM, the trial of interest was modelled as one regressor, and all the other trials were collapsed into five distinct regressors corresponding to recall, miss, false alarms, correction rejection and no response conditions (see Supplementary Table 2 for behavioural performances on the recall task). This approach has been promoted for designs with short interstimulus intervals, when there is a high level of collinearity between haemodynamic responses to successive trials ${ }^{51}$. Further regressors of no interest were the six realignment parameters to account for linear residual motion artefacts. Autocorrelation between the GLM residuals was corrected using the first-order autoregressive process, resulting in prewhitened data after restricted maximum likelihood estimation.

Regions of interest. The MPFC was defined anatomically using the Automated Anatomical Labeling atlas ${ }^{52}$ and was split into vmPFC and dmPFC ROls. The dmPFC corresponded to the bilateral frontal superior medial gyrus of the Automated Anatomical Labeling atlas (indexes 2601 and 2602). The vmPFC mask included the bilateral fronto-orbital medial gyrus (indexes 2611 and 2612), the bilateral rectus (indexes 2701 and 2702) and the ventral portion ( $Z$ coordinates inferior or equal to zero) of the bilateral anterior cingulum (indexes 4001 and 4002). These two ROls are shown in Fig. $3 b$. These two mask images were then wrapped back to each participant's native space using the inverse of the deformation field computed during the normalization process.

Representational similarity analysis. Contrast maps of individual memories were then computed for each recalled picture and used to compute RDMs in our ROls. For each individual and each ROI, brain RDMs were computed as follows: for each voxel, the vector of activity across recalled pictures was mean-centred and scaled to its standard deviation (that is, $z$ score); then, for each pair of pictures, the activity patterns in a given ROI were compared using spatial correlation, and the dissimilarity was then given by 1 minus the correlation. At the behavioural level, individual RDMs were derived from the Euclidean distances between all possible pairs of pictures arranged by the participants on the two-dimensional spatial layout. The upper triangular forms of these brain or behavioural RDMs were then extracted and compared with the upper triangular forms of RDM models describing collective schemas, semantic distances (derived from Wikipedia World War II articles; see below), contextual spatial distances (Euclidean distances of the pictures' spatial positions) and temporal distances (Euclidean distances of the pictures' temporal rank order during Memorial exploration). These comparisons between brain/behavioural and model RDMs were achieved using a regression model. Both regressors and data were initially rank-transformed to test for nonlinear monotonic relationships. Given that collective, semantic (that is, Wikipedia) and contextual (that is, spatial and temporal) model RDMs are not orthogonal and overlap to some extent, a regression model helps clarify the unique variance attributable to each of the model predictors. For each participant, this regression model was reiterated for each topic of interest, and the ten repetitions of the topic model and model regression coefficient were averaged across these iterations. All regression models were full-rank, and the variance inflation factor was less than 1.5 for each regressor, confirming the identifiability and efficiency of our models. The results of these regression models are reported in main text, but we also report the results of standard Spearman's correlations tested in isolation, for the sake of completeness, in Fig. $3 \mathrm{c}$ and in Supplementary Table 1 for statistical tests. Only items correctly recalled were included in the analysis of activity patterns. Grouplevel inferences were conducted using non-parametric randomeffects statistics to test for both RDM relatedness and differences by bootstrapping the subject set with 5,000 iterations ${ }^{28}$. For each model RDM or each pair of contrasted model RDMs, we did not have assumptions about the underlying distribution and performed nonparametric random-effects statistical tests using a bootstrapping approach. We performed a mean comparison at each bootstrap set and estimated the $P$ value as the proportion of bootstrap samples further in the tails than zero. The expected proportions of type I errors across multiple tests of both RDM model relatedness and model comparison were controlled using the FDR correction, with a desired FDR $q=0.05$ and assuming a positive dependency between conditions ${ }^{34[, 53}$. For the test of RDM model relatedness, the expected FDR was computed using all the uncorrected one-tailed $P$ values of tested models. For the test of RDM model comparison, we restricted the correction to our main hypothesis and only included comparisons involving the collective memory RDM (with respect to other reference models) to compute the expected FDR, using twotailed $P$ values. We report adjusted $P$ values and use bootstrapping iterations to determine $95 \%$ percentile Cls. The noise ceiling reported in Fig. $3 \mathrm{c}$ reflects the between-participant correlation of the brain RDMs. This correlation was computed for each participant as the correlation between that participant's brain RDM and the average brain RDM of the remaining participants ${ }^{34}$. The noise ceiling depicted in Fig. $3 c$ corresponds to the average of these individual correlations.

Searchlight analysis. Meshes of the white matter and pial surfaces of the cortex were reconstructed from T1-weighted images collected for each participant using the Freesurfer software package version $5^{54,55}$. We used the rsa_defineSearchlight MATLAB function from the RSA toolbox ${ }^{34}$ (https://github.com/rsagroup/ rsatoolbox), which also relies on Surfing toolbox functions ${ }^{56}$ (https://github.com/ nno/surfing), to define a surface-based searchlight for both hemispheres (using a 40-voxel searchlight with a radius of $10 \mathrm{~mm}$ ). Voxels in the neighbourhood of one or more nodes of the surface were selected using a geodesic distance measure and by constructing virtual lines that connected corresponding nodes on the pial and white matter surfaces. This procedure produces a searchlight following the surface curvature, thus reducing spatial bias during the analysis of $\mathrm{fMRI}$ patterns. In contrast to neighbourhoods defined volumetrically, this resulted in neighbourhoods with a curved cylindrical shape that followed the contours of the sulci and gyri of each individual. Once the searchlight structure for every vertex was constructed and mapped to the functional image space, patterns of recalled activity at each voxel composing the searchlight were mean-centred and scaled to their standard deviations before computing the dissimilarity structure (1 minus the spatial correlation) across all pairwise comparisons of recalled patterns. The upper triangular forms of these searchlight RDMs were then extracted, rank-transformed, and compared using a regression model to the rank-transformed upper triangular form of all RDM models (collective, semantic and contextual). The result of this searchlight analysis created a beta map, a volume in which each voxel contains a statistic for the searchlight centred at that voxel. These first-level beta maps for each model were normalized to the Montreal Neurological Institute T1 template and smoothed using a $10 \mathrm{~mm}$ full-width at half-maximum Gaussian kernel. These standard- 
space participant beta maps were submitted to a second-level nonparametric random-effects analysis in FSL version 5.0.1157. To correct for multiple comparisons, the group-level beta map was submitted to maximal permutation testing using threshold-free cluster enhancement ${ }^{58}$ (TFCE), which offers a good compromise between the overly sensitive cluster-based thresholding and the too conservative whole-brain voxel-based correction. To test RDM model relatedness and differences, TFCE maps were then corrected $\left(P_{\text {corrected }}<0.05\right)$ for the familywise error rate using standard permutation tests implemented in FSL with the randomize function $(10,000$ permutations). The results of the searchlight analysis are reported in Extended Data Fig. 1.

\section{Collective memory corpus description and analysis. The corpus was collected by the MATRICE project} (http://www.matricememory.fr/?lang=en), a multidisciplinary and technological platform, whose aim is to provide tools and technological and theoretical background to understand the relationship between collective and individual memory. The audiovisual clips initially composing the corpus were stored at the National Audiovisual Institute, one of the main partners of the MATRICE project and a public institute whose goal is to archive all audio-visual productions broadcast on French television or radio. For this study, we included in our corpus all television news bulletins and reports (not including radio shows or documentaries) broadcast from 1980 to 2010 with World War II as the common theme, leading to a total of 3,766 documents. We focus on this particular time period for three reasons. First, 1980 to 2010 largely overlaps with the lifetimes of our participants. Second, this period corresponds to the establishment of a new narrative for the French collective memory (that is, a new "Régime of Mémorialité"59). This included the emergence and affirmation of the Shoah memory, as well as major trials acknowledging the participation of the French state and its people, and their representatives at the time (for example, Bousquet, Leguay, Touvier and Papon), in the deportation and killing of Jews. Third, thanks to advances in automatic speech recognition and the availability of electronic texts with which contemporaneous language models were built to process data recorded after 1980, the 3,766 audio files were converted to XML format using speech-to-text conversion algorithms developed by the Laboratoire d'Informatique pour la Mécanique et les Sciences de I'Ingénieur (LIMSI; Engineering Sciences and Computer Science Lab), one of the main partners of the MATRICE project.

Once converted to text, our corpus was initially manually processed to discard segments not related to World War II (exclusively keeping sections dedicated to this topic). During this operation, the automatic speech-to-text transcriptions were additionally corrected by a human reader. After this initial preprocessing, a separate XML file was extracted for each document. These files were then processed with textual data analysis methodologies using the TXM software 60 (http://textometrie.ens-lyon.fr/) connected with the TreeTagger morphosyntactic analyser61 (http://www.cis.unimuenchen.de/ schmid/tools/ TreeTagger/). TXM allows the user to annotate each instance of a word (that is, token) to its corresponding lemma (the canonical form of a set of words) and to proceed to a morphosyntactic labelling of each word in a corpus. The corpus can then be explored using Corpus Query Language (CQL) requests to tag and retrieve specific grammatical categories, forms, lemmas or any information attached to the word. A list of $C Q L$ requests was first automatically generated for each noun, verb or adjective lemma in the corpus. Rare lemmas (less than ten occurrences), stop words and common verbs (for example, "be" and "have") were removed in the process and not included in subsequent analyses. For instance, this algorithm would go through the corpus and produce a unique CQL query (for example, (lemma $=$ "fight"\%c)) corresponding to any instance of the verb "fight" in the corpus (that is, "fight", "fought" or "fighting"). The \%c modifier incorporated in this request is used to neutralize the character case of assimilated lemma forms (that is, "fight", "Fight" or "FIGHT"). An initial list of about 6,500 CQL requests was automatically generated in this way. This list was manually checked to remove CQL requests produced by incorrect transcription, to occasionally group certain requests under a single entity (for example, $($ lemma $=$ "drama"\%c) $\mid($ lemma $=$ "dramatic"\%c) $)$, to merge bigrams when appropriate (for example, (lemma = "chief"\%c)()?(lemma = "state"\%c) for "chief of state") or to adjust to different orthographical forms (for example, (lemma = "Gorbatchev"\%c) $\mid($ lemma = "Gorbatchov"\%c)). After this initial check and adjustment, a total of 6,240 requests remained to summarize the $1,431,790$ tokens composing our corpus.

A lemma $x$ document frequency matrix counting the number of occurrences was extracted and submitted to an LDA analysis performed using the Machine Learning for LanguagE Toolkit (MALLET; http://mallet.cs.umass.edu/topics.php).

Topic models ${ }^{26,29,30}$ consider that documents are generated by mixtures of topics. In this context, a topic corresponds to the distribution of probabilities over all words present across all documents (how likely is a given word to be associated with a topic). From this perspective, a document can be generated by assigning a probability distribution over topics. For each instance in a document, a topic is chosen depending on its previous topic probability distribution, and a word is drawn from that topic. MALLET uses the Gibbs sampling algorithm to invert this process, inferring the set of topics that were responsible for generating a collection of documents and their probabilities over words.

We first trained topic models on the World War II news bulletins and reports corpus using the MALLET train-topic engine (see Extended Data Fig. 2 for an illustration of the topics created with this technique). We varied the number of topics allowed from 2 to 100 , in increments of 1 , setting the alpha parameter to $50 / N$ topics and the starting beta parameter to 0.1 (as suggested in other works modelling a large corpus of texts for semantic purposes ${ }^{62}$ ). For each number of topics, we used 500 iterations to estimate the word and document topic probabilities. We then used the MALLET inferencer tool to fit the LDA model to the Memorial pictures and estimate their topic probabilities. For that purpose, each image was treated as a new document and labelled with keywords (also lemmatized), which were directly derived from the Memorial captions underneath the pictures. A total of $\mathbf{4 4 9}$ lemmas was used to describe the Memorial pictures. Of these lemmas, 428 were also found in the list of 6,240 lemmas describing the corpus of news bulletins and reports. The topic inferential process thus led to a probability distribution matrix of 119 images $\times N$ topics, describing the posterior probability of a topic given an image.

A 119 image $x 119$ image RDM was then computed for each number of estimated topics using the distances between the distributions of topic probabilities for each pair of pictures (based here on the cosine distance, which provides a symmetrical measure of the similarity between two topic vectors). However, given the randomness that the Gibbs sampling algorithm may introduce during parameter estimation, we reiterated the whole process ten times, leading to a 3D 119 image $x 119$ image $\times 10$ repetition RDM for each number of estimated topics. The measurements of the similarity between brain or behavioural RDMs and collective RDMs (beta coefficients of the regression model or Spearman's correlation coefficients) were averaged across those ten repetitions of topic modelling. To summarize the whole process, for a given number of topics: (1) we trained a topic model on the French television news bulletins and reports corpus; (2) we fit this topic model to the Memorial pictures and their captions, treating each picture as a new document; (3) a 119 picture $\times N$ topic matrix, describing the posterior probability of a topic given an image, was extracted and transformed to a $119 \times 119 \mathrm{RDM}$; and (4) this process was reiterated ten times, and for each topic number, the average similarity with brain or behavioural RDMs was computed across those ten instances. 
Validation of the collective memory model and selection of the topic number. We sought to quantify the structure of shared representations across individuals and compared such shared schemas with our collective memory model. This procedure would also permit us to independently select an optimal number of topics to describe the collective structure of pictures that best corresponds to the shared memory. To this end, 54 native French speakers ( 23 males, 31 females) between 20 and 39 years old ( mean $=27.3$ years; s.d. = 5.6) performed the image arrangement task. This task was performed on the exact same 119 Memorial pictures, but unlike our MRI participants, who had visited the Memorial the day before, these new participants were completely unfamiliar with the Memorial. We derived 54 individual RDMs from the Euclidean distances between the images. We then performed DISTATIS ${ }^{33}$ to capture the shared structure of representations across those control individuals. DISTATIS is ideally suited to compute the best agreement or compromise across multiple distance matrices. The implementation in MATLAB of DISTATIS can be found at https://www.utdallas.edu/ herve/, but in brief: (1) each of the 54 RDMs was first transformed to a cross-product matrix after doublecentring and normalization to its first eigenvalue; (2) the cosine similarity structure of all pairwise comparisons of the 54 normalized cross-product matrices was computed using the RV coefficient; (3) the RV coefficient matrix describing the relationships between RDMs was submitted to eigen-decomposition, and the compromise matrix corresponded to the sum of the normalized cross-product matrices weighted by their first eigenvalue; (4) the eigen-decomposition of the compromise produced factor scores, which described the position of each of the 119 images in an $\mathrm{N}$-dimensional compromise space; and (5) the distance correlation matrix of these multidimensional compromise spaces then corresponded to the best agreement across all 54 individual RDMs derived from the behavioural image arrangement task. This distance correlation matrix can be viewed as a shared schema reflecting the common semantic organization across individuals. The collective RDMs extracted from the corpus of television news bulletins and reports were similar to the structure of shared memory measured across control individuals (Fig. 2c). This similarity between collective and shared memory was averaged by bins of five topics, and reached its maximum when six to ten topics were included during topic discovery. As a result, all subsequent analyses involving collective or semantic memory were performed using six to ten topics (and measures of similarity between brain or behavioural RDMs and collective or semantic RDMs were averaged within this selected number of topics).

Construction of a control model of the World War II semantic domain. We used French Wikipedia articles referring to World War II as a benchmark model of the specific semantic relationships between words related to World War II, and trained a topic model that we then fit to the Memorial pictures. This corpus (http://redac. univ-tlse2.fr/corpus/wikipedia.html) included 664,982 articles edited until June 2008, among which 2,643 articles were specifically related to World War II, and has been previously extracted from the dump French version of Wikipedia articles (http://dumps.wikimedia.org/) and processed using the same morphosyntactic tagging tool 61 used to process our corpus of French television news on World War II. Once we had imported the corpus into TXM, we applied exactly the same method of analysis that we previously used to construct the model of collective memory (see Collective memory corpus description and analysis).

Reporting Summary. Further information on research design is available in the Nature Research Reporting Summary linked to this article.

\section{Data availability}

All raw behavioural and imaging data are archived at the GIP Cyceron Centre in
Caen. The collective memory corpus is archived at the INAthèque (Bibliothèque Nationale de France) in Paris, which has the legal deposit. This corpus was collected by the MATRICE project (http://www.matricememory.fr/?lang=en), a multidisciplinary and technological consortium of research units, whose aim is to provide tools and technological and theoretical background to understand the relationship between collective and individual memory. Scientists interested in the analysis of this corpus are welcome to submit a project to the MATRICE project and join the consortium. The tagged Wikipedia corpus used for these analyses is available at http://redac.univ-tlse2.fr/corpus/wikipedia.html.

\section{Code availability}

The text processing and topic analysis of the collective memory and Wikipedia corpora were conducted using TXM (http://textometrie.ens-lyon.fr/) and MALLET (http://mallet.cs.umass.edu/topics.php) software. The MATLAB implementation based on SPM12 (https://www.fil.ion.ucl.ac.uk/spm/) and the RSA toolbox (https:// github.com/rsagroup/rsatoolbox) of the first level and RSAs are available from the corresponding author on request.

\section{References}

1. Halbwachs, M. Les Cadres Sociaux de la Mémoire (Presses Univ. de France, 1925).

2. Bartlett, F. C. Remembering: A Study in Experimental and Social Psychology (Cambridge Univ. Press, 1932).

3. Wertsch, J. V. \& Roediger, H. L. III Collective memory: conceptual foundations and theoretical approaches. Memory 16, 318-326 (2008).

4. Hirst, W. \& Manier, D. Towards a psychology of collective memory. Memory 16, 183-200 (2008).

5. García-Gavilanes, R., Mollgaard, A., Tsvetkova, M. \& Yasseri, T. The memory remains: understanding collective memory in the digital age. Sci. Adv. 3 e1602368 (2017).

6. Olick, J. K. Collective memory: the two cultures. Sociol. Theory 7, 333-348 (1999).

7. Coman, A., Momennejad, I., Drach, R. D. \& Geana, A. Mnemonic convergence in social networks: the emergent properties of cognition at a collective level. Proc. Natl Acad. Sci. USA 113, 8171-8176 (2016).

8. Hirst, W. \& Echterhoff, G. Remembering in conversations: the social sharing and reshaping of memories. Annu. Rev. Psychol. 63, 55-79 (2012).

9. Hirst, W., Yamashiro, J. K. \& Coman, A. Collective memory from a psychological perspective. Trends Cogn. Sci. 22, 438-451 (2018).

10. Gilboa, A. \& Malartte, H. Neurobiology of schemas and schema-mediated memory. Trends Cogn. Sci. 21, 618-631 (2019).

11. Mar, R. M. The neural bases of social cognition and story comprehension. Annu. Rev. Psychol. 62, 103-134 (2011).

12. Krueger, F., Barbey, A. K. \& Grafman, J. The medial prefrontal cortex mediates social event knowledge. Trends Cogn. Sci. 13, 103-109 (2009).

13. van Kesteren, M. T., Fernández, G., Norris, D. G. \& Hermans, E. J. Persistent schema-dependent hippocampal-neocortical connectivity during memory encoding and postencoding rest in humans. Proc. Natl Acad. Sci. USA 107, 7550-7555 (2010)

14. Benoit, R. G., Szpunar, K. K. \& Schacter, D. L. Ventromedial prefrontal cortex supports affective future simulation by integrating distributed knowledge. Proc. Natl Acad. Sci. USA 111, 16550-16555 (2014).

15. Barron, H. C., Dolan, R. J. \& Behrens, T. E. Online evaluation of novel choices by simultaneous representation of multiple memories. Nat. Neurosci. 16, 1492-1498 (2013).

16. Euston, D. R., Gruber, A. J. \& McNaughton, B. L. The role of medial prefrontal cortex in memory and decision making. Neuron 76, 1057-1070 (2012).

17. Wagner, D., Haxby, J. V. \& Heatherton, T. F. The representation of self and person knowledge in the medial prefrontal cortex. WIRES Cogn. Sci. 3, 451470 (2012).

18. Wagner, D., Kelley, W. M., Haxby, J. V. \& Heatherton, T. F. The dorsal medial prefrontal cortex responds preferentially to social interactions during natural viewing. J. Neurosci. 36, 6917-6925 (2016).

19. Van Overwalle, F. Social cognition and the brain: a meta-analysis. Hum. Brain Mapp. 30, 829-858 (2009).

20. Powers, K. E., Chavez, R. S. \& Heatherton, T. F. Individual differences in response of dorsomedial prefrontal cortex predict daily social behavior. Soc. Cogn. Affect. Neurosci. 11, 121-126 (2016).

21. Contreras, J. M., Schirmer, J., Banaji, M. R. \& Mitchell, J. P. Common brain regions with distinct patterns of neural responses during mentalizing about groups and individuals. J. Cogn. Neurosci. 25, 1406-1417 (2013).

22. Chen, J. et al. Shared memories reveal shared structure in neural activity across individuals. Nat. Neurosci. 20, 115-125 (2017).

23. Foltz, P. W. \& Wells, A. D. Automatically deriving readers' knowledge structures from texts. Behav. Res. Methods Instrum. Comput. 31, 208-214 (1999).

24. Manning, J. R., Sperling, M. R., Sharan, A., Rosenberg, E. A. \& Kahana, M. J. Spontaneously reactivated patterns in frontal and temporal lobe predict semantic clustering during memory search. J. Neurosci. 32, 8871-8878 (2012). 
25. Kenett, Y. N., Levi, E., Anaki, D. \& Faust, M. The semantic distance task: quantifying semantic distance with semantic network path length. J. Exp. Psychol. Learn. Mem. Cogn. 43, 1470-1489 (2017).

26. Griffiths, T. L., Steyvers, M. \& Tenenbaum, J. B. Topics in semantic representation. Psychol. Rev. 114, 211-244 (2007).

27. Pereira, F., Detre, G. \& Botvinick, M. Generating text from functional brain images. Front. Hum. Neurosci. 5, 72 (2011).

28. Huth, A. G., de Heer, W. A., Griffiths, T. L., Theunissen, F. E. \& Gallant, J. L. Natural speech reveals the semantic maps that tile human cerebral cortex. Nature 532, 453-458 (2016).

29. Blei, D. M., Ng, A. Y. \& Jordan, M. I. Latent Dirichlet allocation. J. Mach. Learn. Res. 3, 993-1022 (2003).

30. Steyvers, M. \& Griffiths, T. L. in Handbook of Latent Semantic Analysis (eds Landauer, T. et al.) 427-448 (Erlbaum, 2007).

31. Coman, A., Brown, A. D., Koppel, J. \& Hirst, W. Collective memory from a psychological perspective. Int. J. Polit. Cult. Soc. 22, 125-141 (2009).

32. Kriegeskorte, N. \& Mur, M. Inverse MDS: inferring dissimilarity structure from multiple item arrangements. Front. Psychol. 3, 245 (2012).

33. Abdi, H., Williams, L. J., Valentin, D. \& Bennani-Dosse, M. STATIS and DISTATIS: optimum multi-table principal component analysis and three way metric multidimensional scaling. WIRES Comput. Stat. 4, 124-167 (2012).

34. Nili, H. et al. Toolbox for representational similarity analysis. PLoS Comput. Biol. 10, e1003553 (2014).

35. Ferron, M. \& Massa, P. Beyond the encyclopedia: collective memories in Wikipedia. Mem. Stud. 7, 22-45 (2014).

36. Pentzold, C. Fixing the floating gap: the online encyclopaedia Wikipedia as a global memory place. Mem. Stud. 2, 255-272 (2009).

37. Chikazoe, J., Lee, D. H., Kriegeskorte, N. \& Anderson, A. K. Population coding of affect across stimuli, modalities and individuals. Nat. Neurosci. 17, 11141122 (2014).

38. Bracci, S., Caramazza, A. \& Peelen, M. V. Representational similarity of body parts in human occipitotemporal cortex. J. Neurosci. 35, 12977-12985 (2015)

39. Brod, G., Lindenberger, U., Werkle-Bergner, M. \& Shing, Y. L. Differences in the neural signature of remembering schema-congruent and schemaincongruent events. Neuroimage 117, 358-366 (2015).

40. Pernet, C. R., Wilcox, R. \& Rousselet, G. A. Robust correlation analyses: false positive and power validation using a new open source MATLAB toolbox. Front. Psychol. 3, 606 (2013).

41. Kumaran, D., Summerfield, J. J., Hassabis, D. \& Maguire, E. A. Tracking the emergence of conceptual knowledge during human decision making. Neuron 63, 889-901 (2009).

42. Edelson, M., Sharot, T., Dolan, R. J. \& Dudai, Y. Following the crowd: brain substrates of long-term memory conformity. Science 333, 108-111 (2011).

43. Echterhoff, G., Higgins, E. T. \& Levine, J. M. Shared reality: experiencing commonality with others' inner states about the world. Psychol. Sci. 4, 496521 (2009).

44. Nicolle, A. et al. An agent independent axis for executed and modeled choice in medial prefrontal cortex. Neuron 75, 1114-1121 (2012).

45. Friston, K. \& Frith, C. A duet for one. Conscious. Cogn. 36, 390-405 (2015)

46. Mahr, J. \& Csibra, G. Why do we remember? The communicative function of episodic memory. Behav. Brain Sci. e1, 1-63 (2017).

47. Constantinescu, A. O., O'Reilly, J. X. \& Behrens, T. E. J. Organizing conceptua knowledge in humans with a gridlike code. Science 352, 1464-1468 (2016).

48. Yun, J., Lee, S. H. \& Jeong, H. Early onset of structural inequality in the formation of collaborative knowledge in all Wikimedia projects. Nat. Hum. Behav. 3, 155-163 (2019).

49. Peschanski, D. in Mémoire et Oubli (ed. Eustache, F.) 71-91 (Le Pommier, 2014).

50. Turner, B. O., Mumford, J. A., Poldrack, R. A. \& Ashby, F. G. Spatiotemporal activity estimation for multivoxel pattern analysis with rapid event-related designs. Neurolmage 62, 1429-1438 (2012).

51. Mumford, J. A., Turner, B. O., Ashby, F. G. \& Poldrack, R. A. Deconvolving BOLD activation in event-related designs for multivoxel pattern classification analyses. Neurolmage 59, 2636-2643 (2012).

52. Tzourio-Mazoyer, N. et al. Automated anatomical labeling of activations in SPM using a macroscopic anatomical parcellation of the MNI MRI singlesubject brain. Neurolmage 15, 273-289 (2002).

53. Benjamini, Y. \& Yekutieli, D. The control of the false discovery rate in multiple testing under dependency. Ann. Stat. 29, 1165-1188 (2001).

54. Dale, A. M., Fischl, B. \& Sereno, M. I. Cortical surface-based analysis. I. Segmentation and surface reconstruction. Neurolmage 9, 179-194 (1999).

55. Fischl, B., Sereno, M. I. \& Dale, A. M. Cortical surface-based analysis. II. Inflation, flattening, and a surface-based coordinate system. Neurolmage $\mathbf{9}$, 195-207 (1999).

56. Oosterhof, M. N., Wiesterl, T., Downing, P. E. \& Diedrichsen, J. A comparison of volume-based and surface-based multi-voxel pattern analysis. Neurolmage 56, 593-600 (2011).

57. Winkler, A. M., Ridgway, G. R., Webster, M. A., Smith, S. \& Nichols, T. E. Permutation inference for the general linear model. Neurolmage 92, 381397 (2014).
58. Smith, S. M. \& Nichols, T. E. Threshold-free cluster enhancement: addressing problems of smoothing, threshold dependence and localisation in cluster inference. Neurolmage 44, 83-98 (2009).

59. Peschanski, D. Les Années Noires 387-402 (Hermann, 2012).

60 . Heiden, S. The TXM platform: building open-source textual analysis software compatible with the TEI encoding scheme. In 24th Pacific Asia Conference on Language, Information and Computation (eds Otoguro, R. et al.) 389-398 (Institute for Digital Enhancement of Cognitive Development, Waseda University, 2010)

61. Schmid, H. Probabilistic part-of-speech tagging using decision trees. In International Conference on New Methods in Language Processing 44-49 (1994).

62. Griffiths, T. L. \& Steyvers, M. Finding scientific topics. Proc. Natl Acad. Sci. USA 101, 5228-5235 (2004).

\section{Acknowledgements}

We thank R. Benoit and M. Wimber for helpful corrections and comments, as well as $\mathrm{R}$. Henson for helpful discussions, on an earlier version of this manuscript. We thank B. Pincemin for her help in the early stages of this project, as well as for her final comments on this manuscript. We thank S. Grimaldi, head of the Caen Memorial, for giving us full access and clearance to proceed with our experiment. We thank "I'observatoire B2V des Mémoires" and M. Morel for help with data collection for the image arrangement task, as well as $\mathrm{H}$. Abdi for guidance on data analysis. We thank $\mathrm{D}$. Maréchal for his help with the selection of television news, as well as K. Dauchot and $M$ Dufossé for their help during the initial piloting of the experiment. We thank E. Portier and T. Chan for editing the English of the main text. We also thank all participants for volunteering in this study. The study was supported by an EQUIPEX (MATRICE) led by D.P. and funded by the French National Research Agency (grant no. 10-EQPX-21-01), and by a three-year postdoctoral fellowship from the Normandy region to P.G. The funders had no role in study design, data collection and analysis, decision to publish or preparation of the manuscript.

\section{Author contributions}

P.G., D.P. and F.E. designed the experiment and wrote the article. P.G. analysed the data. T.V. designed and programmed the image arrangement task, constructed experimental material, processed the corpus and collected the data. S.H. and M.D. programmed the script embedded in TXM to lexically process the corpus. J.-L.G. and A.L. designed and programmed the speech-to-text conversion algorithm to transform the corpus of news into text. C.K.-P. and D.P. supervised the collection of the collective memory corpus and its storing, manual editing and trimming. F.V. supervised the MRI data collection.

\section{Competing interests Additional information}

The authors declare no competing interests.

Extended data is available for this paper at https://doi.org/10.1038/s41562-019-0779z. Supplementary information is available for this paper at https://doi.org/10.1038/ s41562-019-0779-z.

Correspondence and requests for materials should be addressed to P.G. 


\section{Collective schema}
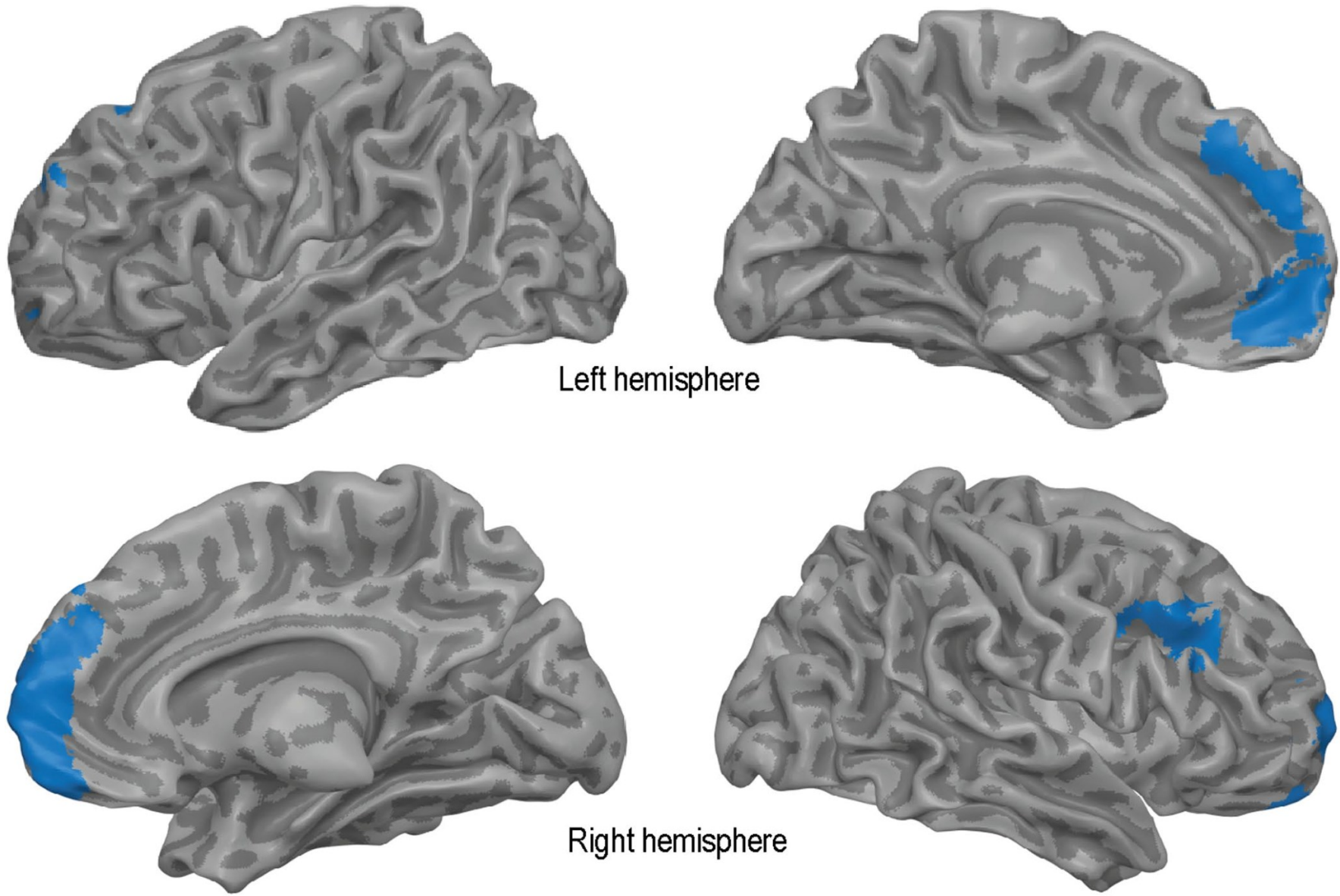

Extended Data Fig. 1 | results of searchlight analysis. A searchlight analysis following the surface curvature of the brain was ran to test the effects of collective schema, and contextual and semantic memory. At each searchlight, brain RDM was extracted and compared to RDM models using a regression model, thus producing a beta map for each participant $(n=24)$ and RDM model. To correct for multiple comparisons, the group-level beta map was submitted to maximal permutation testing using threshold-free cluster enhancement $\left(T F C E^{58}\right)$. Blue regions indicate clusters surviving TFCE correction across the whole brain $(P$ corrected < .05) for collective schema. There was no statistically significant evidence that brain regions were related to other RDM models using the searchlight approach. 


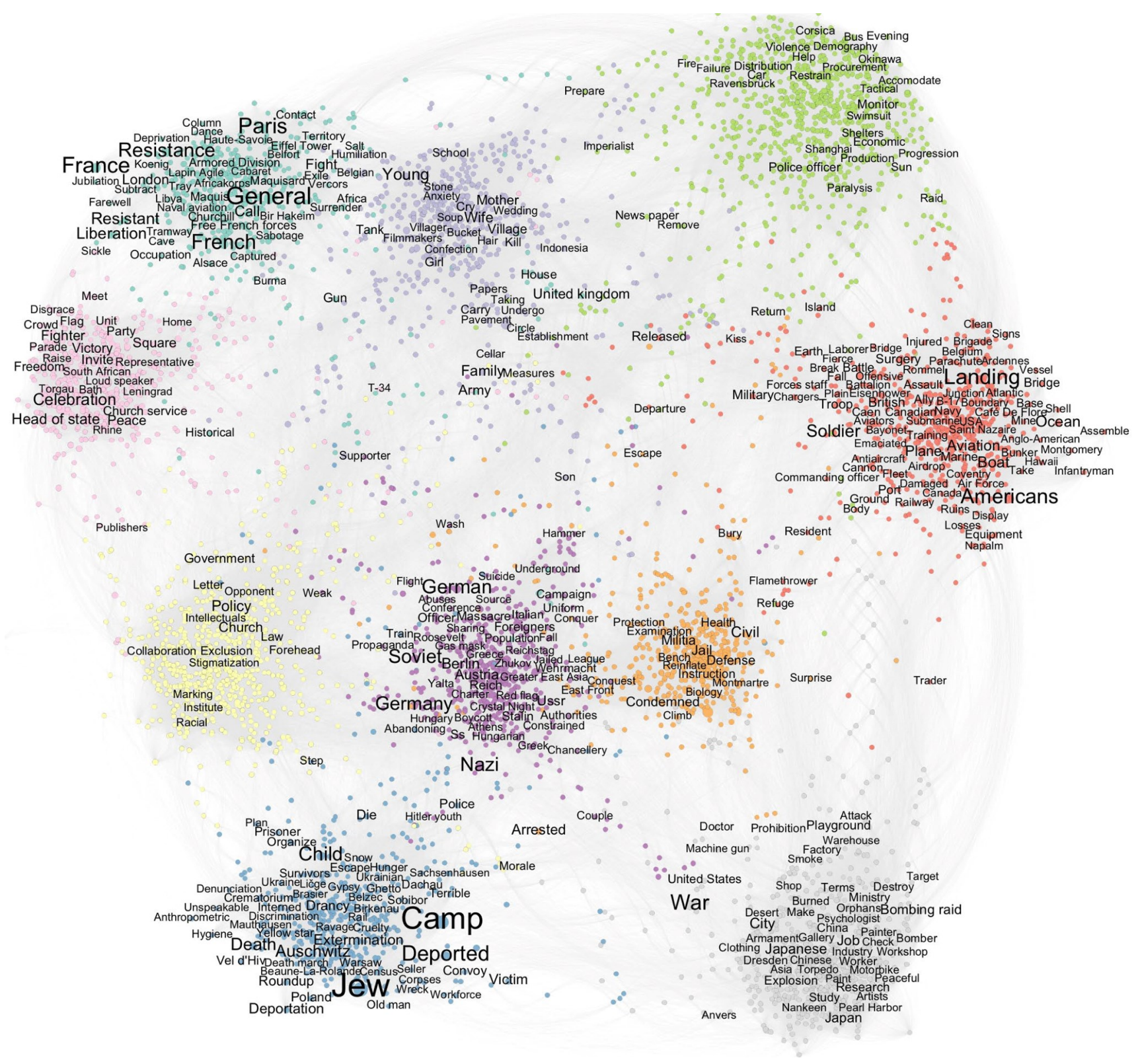

Extended Data Fig. 2 | Graph network of topic model. To illustrate the semantic model and the connections between words derived from our topic models, we computed a Lemmas $x$ Lemmas correlation matrix using the estimated distribution of probabilities over 10 topics. This correlation matrix was then thresholded and transformed into a binary adjacency matrix by keeping the top $10 \%$ of the strongest connections between lemmas. The adjacency matrix is visualized here using a force vector algorithm proposed with the Gephi software (https://gephi.org/). Each node represents one of the 6,240 lemmas. The color and the size of the node is determined by its maximal topic assignment and probability, respectively. Amongst the 6,240 nodes, only key words describing Memorial pictures and specifically translated into English, are displayed here for visualization purpose. The size of the label is proportional to its topic probability. The distribution of topic probabilities for each Memorial pictures is directly derived from the topic probabilities associated with these key words. 